\title{
Moments of Two-Variable Functions and the Uniqueness of Graph Limits
}

\author{
Christian Borgs \\ Jennifer Chayes \\ Microsoft Research, Cambridge, MA \\ László Lovász \\ Institute of Mathematics, Eötvös Loránd University, Budapest, Hungary
}

December 2008

\begin{abstract}
For a symmetric bounded measurable function $W$ on $[0,1]^{2}$ and a simple graph $F$, the homomorphism density

$$
t(F, W)=\int_{[0,1]^{V(F)}} \prod_{i j \in E(F)} W\left(x_{i}, x_{j}\right) d x .
$$

can be thought of as a "moment" of $W$. We prove that every such function is determined by its moments up to a measure preserving transformation of the variables.

The main motivation for this result comes from the theory of convergent graph sequences. A sequence $\left(G_{n}\right)$ of dense graphs is said to be convergent if the probability, $t\left(F, G_{n}\right)$, that a random map from $V\left(G_{n}\right)$ into $V(F)$ is a homomorphism converges for every simple graph $F$. The limiting density can be expressed as $t(F, W)$ for a symmetric bounded measurable function $W$ on $[0,1]^{2}$. Our results imply in particular that the limit of a convergent graph sequence is unique up to measure preserving transformation.
\end{abstract}




\section{Contents}

1 Introduction

2 Results 4

2.1 Graphons and Graph Densities .............. 4

2.2 Main results . . . . . . . . . . . . . . . . . . 6

2.3 Examples ....................... 9

3 Isomorphism 9

3.1 Preliminaries ................... . . 10

3.2 Push-Forward and Quotients . . . . . . . . . . . . . 11

3.3 Reductions ..................... 13

3.3.1 Making a graphon strong . . . . . . . . . . . . . 14

3.3.2 Countable generation . . . . . . . . . . . . . . 14

3.3.3 Merging inseparable elements . . . . . . . . . . . 15

3.3.4 Lebesgue property . . . . . . . . . . . . . . 15

3.3.5 Partitions into Twin-Classes . . . . . . . . . . . . . 15

3.4 Isomorphism and Weak Isomorphism . . . . . . . . . 17

4 Canonical Ensembles $\mathbf{1 8}$

4.1 Measure theoretic preparation . . . . . . . . . . . . 19

4.2 Anchor Sequences . . . . . . . . . . . . . . . . . 22

5 Coupling 23

5.1 Partially Labeled Graphs and Marginals . . . . . . . . . . . . 23

5.2 Multiple Edges . . . . . . . . . . . . . . . . . . . . . . . . . . . . . . . .

5.3 Coupling Anchor Sequences . . . . . . . . . . . 26

5.4 Conclusion of proofs . . . . . . . . . . . . . . 30

6 Appendix: Moments and coupling of probability distributions

33

\section{Introduction}

Let $\mathcal{W}$ be the set of bounded symmetric measurable functions $W:[0,1]^{2} \rightarrow$ $\mathbb{R}$, and let $\mathcal{W}_{0}$ denote the set of functions in $\mathcal{W}$ with values in $[0,1]$. For 
every $W \in \mathcal{W}$ and every finite graph $F$, we define the integral

$$
t(F, W)=\int_{[0,1]^{n}} \prod_{i j \in E(F)} W\left(x_{i}, x_{j}\right) \prod_{i \in V(F)} d x_{i} .
$$

Our interest in these integrals stems from graph theory (see next paragraph), but such integrals appear in physics, statistics, and other areas. In many respects, these integrals can be thought of as 2-variable analogues of moments of 1-variable functions, so instead of moment sequences, such 2-variable functions have a "moment graph parameter" (function defined on graphs). Just like moments of a 1-variable function determine the function up to measure preserving transformations, these "moments" determine the 2-variable function up to measure preserving transformations. The exact formulation and proof of this fact is the main goal of this paper.

Our main motivation for this study comes from the theory of convergent graph sequences. Let $F$ and $G$ be two simple graphs (graphs without loops and multiple edges). Let us map the nodes of $F$ randomly into $V(G)$, and let $t(F, G)$ denote the probability that this map preserves adjacency. For example, $t\left(K_{2}, G\right)$ denotes the edge density of $G$. In general, we call $t(F, G)$ the homomorphism density or simply the density of $F$ in $G$.

We call a sequence of simple graphs $\left(G_{n}\right)$ convergent, if $t\left(F, G_{n}\right)$ has a limit for every simple graph $F$. The notion of convergent graph sequences was introduced by Borgs, Chayes, Lovász, Sós and Vesztergombi [2], see also [3], and further studied in [4] and [5]. Lovász and Szegedy [11] proved that every convergent graph sequence has a "limit object" in the form of a function $W \in \mathcal{W}_{0}$ in the sense that

$$
t\left(F, G_{n}\right) \longrightarrow t(F, W) \quad \text { as } \quad n \rightarrow \infty
$$

for every simple graph $F$. In this case we say that $G_{n}$ converges to $W$. It was also shown in [11] that for every function $W \in \mathcal{W}_{0}$ there is a convergent sequence $\left(G_{n}\right)$ of simple graphs converging to $W$. To complete the picture, the results in this paper imply that the limit object is unique up to measure preserving transformations. 


\section{Results}

For the precise statement of our results, we need some definitions. Instead of the interval $[0,1]$, we consider two-variable functions on an arbitrary probability space; while this does not add real generality it leads to a cleaner picture. We need a few definitions.

We start by recalling some basic notions from probability theory. Let $(\Omega, \mathcal{A}, \pi)$ be a probability space (where $\Omega$ is the underlying set, $\mathcal{A}$ is a $\sigma$ algebra on $\Omega$, and $\pi$ is a probability measure on $\mathcal{A})$. As usual, $(\Omega, \mathcal{A}, \pi)$ is called complete if $\mathcal{A}$ contains all sets of external measure 0 , and the completion of $(\Omega, \mathcal{A}, \pi)$ is obtained by replacing $\mathcal{A}$ with the $\sigma$-algebra generated by $\mathcal{A}$ and all subsets $N \subset \Omega$ of external measure 0 .

Let $(\Omega, \mathcal{A}, \pi)$ and $\left(\Omega^{\prime}, \mathcal{A}^{\prime}, \pi^{\prime}\right)$ be probability spaces, and let $\phi$ be a measure preserving map from $\Omega$ to $\Omega^{\prime}$. The map $\phi$ is called an isomorphism if it is a bijection between $\Omega$ and $\Omega^{\prime}$ and both $\phi$ and $\phi^{-1}$ are measure preserving, and it is called an isomorphism $\bmod 0$ if there are null sets $N \in \mathcal{A}$ and $N^{\prime} \in \mathcal{A}^{\prime}$ such that the restriction of $\phi$ to $\Omega \backslash N$ is an isomorphism between $\Omega \backslash N$ and $\Omega^{\prime} \backslash N^{\prime}$ (equipped with the suitable restrictions of $(\mathcal{A}, \pi)$ and $\left(\mathcal{A}^{\prime}, \pi^{\prime}\right)$, respectively). In the last case $(\Omega, \mathcal{A}, \pi)$ and $\left(\Omega^{\prime}, \mathcal{A}^{\prime}, \pi^{\prime}\right)$ are called isomorphic $\bmod 0$.

It turns out that several of our results require a little bit more structure than that of an arbitrary probability space. In particular, we will consider Lebesgue spaces, i.e., complete probability spaces that are isomorphic mod 0 to the disjoint union of a closed interval (equipped with the standard Lebesgue sets and Lebesgue measure) and a countable set of atoms 1

\subsection{Graphons and Graph Densities}

We are now ready to introduce the main objects studied in this paper.

\footnotetext{
${ }^{1}$ See [13, Section 2.2 for an axiomatic definition of Lebesgue spaces, and Section 2.4 for the proof that a probability space is Lebesgue if and only if it is isomorphic mod 0 to the disjoint union of a closed interval and a countable set of atoms.
} 
Starting from an arbitrary probability space $(\Omega, \mathcal{A}, \pi)$, let $W: \Omega \times \Omega \rightarrow \mathbb{R}$ be a bounded, symmetric function measurable with respect to the completion of $(\Omega \times \Omega, \mathcal{A} \times \mathcal{A}, \pi \times \pi)$. We call the quadruple $H=(\Omega, \mathcal{A}, \pi, W)$ a graphon, and refer to $W$ as a graphon on the probability space $(\Omega, \mathcal{A}, \pi)$. (As discussed above, such functions can be thought of as limits convergent graph sequences, which explains the name).

From our point of view, graphons obtained by changing $W$ on a set of measure 0 , or changing the $\sigma$-algebra $\mathcal{A}$ so that $W$ remains measurable, do not differ essentially from the original. However, for technical reasons we have to distinguish them. We say that a graphon is strong, if $W$ is measurable with respect to $\mathcal{A} \times \mathcal{A}$ (not just the completion of it). We can always change $W$ on a set of measure 0 to make the graphon strong (Theorem $3.2(\mathrm{i})$ ).

We say that $H$ is complete, if the underlying probability space is complete, and we say that it is Lebesguian, if the underlying probability space is a Lebesgue space. The completion, $\bar{H}$, of $H$ is obtained by completing the underlying probability space, i.e., by replacing $\mathcal{A}$ by its completion $\overline{\mathcal{A}}$.

Let $H=(\Omega, \mathcal{A}, \pi, W)$ be a graphon, and let $F$ be a finite graph with $V(F)=\{1, \ldots, k\}$. The definition (1) then can be extended as

$$
t(F, H)=\int_{\Omega^{k}} \prod_{i j \in E(F)} W\left(x_{i}, x_{j}\right) \prod_{i=1}^{k} d \pi\left(x_{i}\right) .
$$

Let $H=(\Omega, \mathcal{A}, \pi, W)$ and $H^{\prime}=\left(\Omega^{\prime}, \mathcal{A}^{\prime}, \pi^{\prime}, W^{\prime}\right)$ be two graphons. The goal of this paper is to determine necessary and sufficient conditions under which

$$
t(F, H)=t\left(F, H^{\prime}\right)
$$

for all graphs $F$.

To this end, we will introduce two different notions of isomorphism. Both will be expressed in terms of the following operation: given a graphon $H^{\prime}=\left(\Omega^{\prime}, \mathcal{A}^{\prime}, \pi^{\prime}, W^{\prime}\right)$ and a measure preserving map $\phi$ from a probability space $(\Omega, \mathcal{A}, W)$ into $\left(\Omega^{\prime}, \mathcal{A}^{\prime}, \pi^{\prime}\right)$, let $\left(W^{\prime}\right)^{\phi}$ be "pull-back" of $W^{\prime}$, defined by $\left(W^{\prime}\right)^{\phi}(x, y)=W(\phi(x), \phi(y))$. If $H=(\Omega, \mathcal{A}, \pi, W)$ and $G=(\Gamma, \mathcal{B}, \rho, U)$ are 
two graphons and $\phi: \Omega \rightarrow$ Gamma is measure preserving from the completion $\overline{\mathcal{A}}$ into $\mathcal{B}$ such that $W=U^{\phi}$ almost everywhere, then we call $\phi$ a weak isomorphism from $H$ to $G$. Note that a weak isomorphism is not necessarily invertible.

We say that $H$ and $H^{\prime}$ are isomorphic $\bmod 0$ (in notation $H^{\prime} \cong H^{\prime}$ ), if there exists a map $\phi: \Omega \rightarrow \Omega^{\prime}$ such that $\phi$ is an isomorphism mod 0 and $\left(W^{\prime}\right)^{\phi}=W$ almost everywhere in $\Omega \times \Omega$. For simplicity, we often drop the qualifier $\bmod 0$.

We call $H$ and $H^{\prime}$ weakly isomorphic if there is a third graphon $G$ and weak isomorphisms from $H$ and $H^{\prime}$ into $G$. It will follow from Theorems 3.2 and 2.1 that we could require here that $G$ is a strong Lebesguian graphon.

The isomorphism relation $\cong$ is clearly an equivalence relation, and it will follow from Theorem 2.1 (ii) below that weak isomorphism is an equivalence relation as well. Every graphon is weakly isomorphic with its completion, and every pair of isomorphic graphons is weakly isomorphic. It is clear that if two graphons $H$ and $H^{\prime}$ are weakly isomorphic then (44) holds for every graph $H$. Theorem 2.1 (ii) below will show that the converse also holds.

To state our results, we need one more notion, the notion of twins. Let $H=(\Omega, \mathcal{A}, \pi, W)$ be a graphon. Two points $x_{1}, x_{2} \in \Omega$ are called twins if $W\left(x_{1}, y\right)=W\left(x_{2}, y\right)$ for almost all $y \in \Omega$. Note that relation of being twins is an equivalence relation. We call the graphon $H$ almost twin-free if all there exists a set $N$ of measure zero such that no two points in $\Omega \backslash N$ are twins.

\subsection{Main results}

With these definitions, we can state our main result:

Theorem 2.1 (i) If $H$ and $H^{\prime}$ are almost twin-free Lebesguian graphons, then (4) holds for every simple graph $F$ if and only if $H \cong H^{\prime}$.

(ii) If $H$ and $H^{\prime}$ are general graphons, then (4) holds for every simple graph $F$ if and only if $H$ and $H^{\prime}$ are weakly isomorphic. 
A natural idea of the proof of Theorem 2.1 is the following: can we bring a graphon $(\Omega, \mathcal{A}, \pi, W)$ to a "canonical form", so that isomorphic or weakly isomorphic graphons would have identical canonical forms? In the case of functions in a single variable, this is possible, through "monotonization": for every bounded real function on $[0,1]$ there is an unique monotone increasing left-continuous function on $[0,1]$ that has the same moments.

In Section 4 we'll construct not quite a canonical form, but a "canonical ensemble", a probability distribution $\left(H_{\alpha}\right)$ of graphons on the same $\sigma$-algebra such that $H \cong H_{\alpha}$ for almost all $\alpha$, and two graphons are isomorphic if and only if their ensembles can be coupled so that corresponding graphons are identical (up to sets of measure 0).

An important element of the proof is a curious measure-theoretic fact. Consider a 2-variable function for which all 1-variable functions obtained by fixing one of the variables are measurable. This of course does not in general imply that the 2-variable function is measurable, but it does imply it in some circumstances (see e.g. Corollary 4.2).

As we will see, the second statement of Theorem 2.1 can easily be deduced from the first. In fact, we'll show that every graphon is weakly isomorphic to a twin-free Lebesguian graphon. (See Theorem 3.2 for more details of this isomorphism.)

We can also transform a Lebesguian graphon into a graphon whose underlying probability space is the unit interval with the Lebesgue measure, by "resolving" the atoms into intervals of the appropriate length. This form is the most elementary and therefore useful in applications; however, it is not so convenient for the purposes of this paper because we loose twin-freeness.

It is easy to see that if $H$ and $H^{\prime}$ are weakly isomorphic, then (44) holds not only for simple graphs $F$ but also for graphs with multiple edges (which we'll call multigraphs if we want to emphasize that multiple edges are allowed; but we don't allow loops). Thus (4) for simple graphs implies this equation for multigraphs. (This fact will be an important step in the proof, see Section 5.2.) 
We can formulate our results in a probabilistic way. Recall that a coupling between two probability spaces $(\Omega, \mathcal{A}, \pi)$ and $\left(\Omega^{\prime}, \mathcal{A}^{\prime}, \pi^{\prime}\right)$ is a probability distribution on $\mathcal{A} \times \mathcal{A}^{\prime}$ whose marginals are $\pi$ and $\pi^{\prime}$, respectively. A coupling between two graphons means a coupling between their underlying probability spaces. Let $H=(\Omega, \mathcal{A}, \pi, W)$ be a graphon, and let $X_{1}, \ldots, X_{n}$ be independent random samples from $\pi$. Then we have

$$
t(F, H)=\mathrm{E}\left(\prod_{i j \in E(F)} W\left(X_{i}, X_{j}\right)\right) .
$$

Let $H=(\Omega, \mathcal{A}, \pi, W)$ and $H^{\prime}=\left(\Omega^{\prime}, \mathcal{A}^{\prime}, \pi^{\prime}, W^{\prime}\right)$ two graphons, and suppose that there exists a coupling $\gamma$ between them such that $W\left(X_{1}, Y_{1}\right)=$ $W^{\prime}\left(X_{2}, Y_{2}\right)$ holds with probability 1 for two independent samples $\left(X_{1}, X_{2}\right)$ and $\left(Y_{1}, Y_{2}\right)$ from $\gamma$. In this case clearly (4) holds for every graph $F$. As we will see, Theorem 2.1 implies that in the Lebesguian case the converse also holds.

We sum up the results for the most important special case of functions in $\mathcal{W}$, i.e., bounded, symmetric functions $W:[0,1]^{2} \rightarrow \mathbb{R}$ which are measurable with respect to the Lebesgue sets on $[0,1]^{2}$ (the Corollary would remain valid for arbitrary Lebesguian graphons, but this would not be essentially more general).

Corollary 2.2 For two functions $W, W^{\prime} \in \mathcal{W}$ the following are equivalent.

(a) For every simple graph $F, t\left(F, W^{\prime}\right)=t(F, W)$.

(b) For every multigraph $F, t\left(F, W^{\prime}\right)=t(F, W)$.

(c) There exists a function $U \in \mathcal{W}$ and two measure preserving maps $\varphi, \psi:[0,1] \rightarrow[0,1]$ such that $W=U^{\varphi}$ and $W^{\prime}=U^{\psi}$ almost everywhere.

(d) There exist two measure preserving maps $\varphi, \psi:[0,1] \rightarrow[0,1]$ such that $\left(W^{\prime}\right)^{\varphi}=W^{\psi}$ almost everywhere.

(e) There exists a probability measure $\gamma$ on $[0,1] \times[0,1]$ such that each marginal of $\gamma$ is the Lebesgue measure, and if $\left(X, X^{\prime}\right)$ and $\left(Y, Y^{\prime}\right)$ are two independent samples from $\gamma$, then $\left.W(X, Y)=W^{\prime}\left(X^{\prime}, Y^{\prime}\right)\right)$ with probability 1. 


\section{$2.3 \quad$ Examples}

The property of being twin-free is crucial for Theorem 2.1 (i).

Example 1 Let $\phi_{k}:[0,1] \rightarrow[0,1]$ be the map $\phi_{k}(x)=k x(\bmod 1)$. For any function $W \in \mathcal{W}$, the functions $W^{\phi_{2}}$ and $W^{\phi_{3}}$ define graphons that are weakly isomorphic but in general not isomorphic. Indeed, for a "generic" $W$ (say $W=x y$ ), every point has two twins in $W^{\phi_{2}}$ and three twins in $W^{\phi_{3}}$. The pair of maps in Corollary 2.2 (c) go from $W$, while in (d), they go into $\left(W^{\phi_{3}}\right)^{\phi_{2}}=\left(W^{\phi_{2}}\right)^{\phi_{3}}=W^{\phi_{6}}$.

Our next example shows that the Lebesgue property is also needed.

Example 2 Let $\Omega$ be a subset of $[0,1]$ with inner Lebesgue measure 0 and outer Lebesgue measure 1 , and let $\Omega^{\prime}$ be its complement. Let $\mathcal{A}$ and $\mathcal{A}^{\prime}$ consist of the traces of Lebesgue measurable sets on $\Omega$ and $\Omega^{\prime}$, respectively. Let $W$ and $W^{\prime}$ be the restrictions of the function $x y$ to $\Omega \times \Omega$ and $\Omega^{\prime} \times \Omega^{\prime}$, respectively. The identical embeddings $\varphi: \Omega \rightarrow[0,1]$ and $\varphi^{\prime}: \Omega^{\prime} \rightarrow[0,1]$ are measure preserving, and hence $H=(\Omega, \mathcal{A}, \pi, W)$ and $H^{\prime}=\left(\Omega^{\prime}, \mathcal{A}^{\prime}, \pi^{\prime}, W^{\prime}\right)$ are weakly isomorphic. But for every $x \in \Omega$, we have

$$
2 \int_{\Omega} W(x, y) d \pi(y)=x \notin \Omega^{\prime},
$$

which shows that there is no way to "match up" the points in $\Omega$ and $\Omega^{\prime}$ to get an isomorphism mod 0 . The same example shows that conclusions (d), (e) in Corollary 2.2 could not be extended to the non-Lebesgue case either.

\section{Isomorphism}

The main goal of this section is to describe how a general graphon can be transformed into a twin-free Lebesguian graphon. To this end, we have to recall some basic notions from measure theory (mostly because their usage does not seem standard), and then discuss different "isomorphism-like" mappings between graphons. 


\subsection{Preliminaries}

For a set $\mathcal{S}$ of subsets of a set $\Omega$, we denote by $\sigma(\mathcal{S})$ the $\sigma$-algebra generated by $\mathcal{S}$. We call a $\sigma$-algebra $\mathcal{A}$ countably generated if there is countable set $S \subseteq \mathcal{A}$ such that $\sigma(\mathcal{S})=\mathcal{A}$. This is equivalent to the existence of a sequence $\mathcal{A}_{1} \subseteq \mathcal{A}_{2} \subseteq \ldots$ of finite $\sigma$-algebras whose union generates $\mathcal{A}$.

We say that a set $\mathcal{S} \subseteq \mathcal{A}$ is a basis for the probability space $(\Omega, \mathcal{A}, \pi)$, if $\sigma(S)$ is dense in $\mathcal{A}$, i.e., for every $X \in \mathcal{A}$ there is a $Y \in \sigma(\mathcal{S})$ such that $\pi(X \triangle Y)=0$.

Given sets $A \subset \Omega$ and two points $x, y \in \Omega$, we say that $A$ separates $x$ and $y$ if $|\{x, y\} \cap A|=1$. We say that a set $\mathcal{S}$ of subsets of $\Omega$ separates $x$ and $y$ if there exists a set $A \in \mathcal{S}$ that separates $x$ and $y$. This leads to a partition $\mathcal{P}[\mathcal{S}]$ of $\Omega$ by placing two points in the same class if and only if they are not separated by $\mathcal{S}$. We say that $\mathcal{S}$ is separating if it separates any two points in $\Omega$. We'll say that a graphon is separating if its underlying $\sigma$-algebra is separating.

A probability space $\left(\Omega^{\prime}, \mathcal{A}^{\prime}, \pi^{\prime}\right)$ is called a full subspace of $(\Omega, \mathcal{A}, \pi)$ if $\Omega^{\prime}$ is a (not necessarily measurable) subset of $\Omega$ of external measure $1, \mathcal{A}^{\prime}=$ $\left\{A \cap \Omega^{\prime} \mid A \in \mathcal{A}\right\}$, and $\left.\pi^{\prime}\left(A \cap \Omega^{\prime}\right)\right)=\pi(A)$ for all $A \in \mathcal{A}$.

Consider two probability spaces $(\Omega, \mathcal{A}, \pi)$ and $\left(\Omega^{\prime}, \mathcal{A}^{\prime}, \pi^{\prime}\right)$ and a measure preserving map $\phi: \Omega \rightarrow \Omega^{\prime}$. The map $\phi$ is called an embedding of the first space into the second if $\phi$ is an isomorphism between $(\Omega, \mathcal{A}, \pi)$ and a full subspace of $\left(\Omega^{\prime}, \mathcal{A}^{\prime}, \pi^{\prime}\right)$. We call $\phi$ an embedding of a graphon $H=$ $(\Omega, \mathcal{A}, \pi, W)$ into a graphon $H^{\prime}=\left(\Omega^{\prime}, \mathcal{A}^{\prime}, \pi^{\prime}, W^{\prime}\right)$ if $\phi$ is an embedding of $(\Omega, \mathcal{A}, \pi)$ into $\left(\Omega^{\prime}, \mathcal{A}^{\prime}, \pi^{\prime}\right)$ and $\left(W^{\prime}\right)^{\phi}=W$ almost everywhere.

Let $(\Omega, \mathcal{A}, \pi)$ be a probability space and $f: \Omega \rightarrow \mathbb{R}$, a bounded $\mathcal{A}$ measurable function. Let $\mathcal{A}_{0} \subseteq \mathcal{A}$ be a sub- $\sigma$-algebra. The conditional expectation $\mathrm{E}\left(f \mid \mathcal{A}_{0}\right)$ is the set of all $\mathcal{A}_{0}$-measurable function $f^{\prime}$ such that

$\int_{A_{0}} f d \pi=\int_{A_{0}} f^{\prime} d \pi$ for all $A_{0} \in \mathcal{A}_{0}$. It is well known that such functions exist and any two such functions differ only on a set of $\pi$-measure 0 . We'll write (somewhat sloppily) $f^{\prime}=\mathrm{E}\left(f \mid \mathcal{A}_{0}\right)$ instead of $f^{\prime} \in \mathrm{E}\left(f \mid \mathcal{A}_{0}\right)$. We say that $f$ is almost $\mathcal{A}_{0}$-measurable, if there is an $\mathcal{A}_{0}$-measurable function $f^{\prime}$ such 
that $f=f^{\prime} \pi$-almost everywhere. Clearly we must have $f^{\prime} \in \mathrm{E}\left(f \mid \mathcal{A}_{0}\right)$, and it does not matter which representative of $\mathrm{E}\left(f \mid \mathcal{A}_{0}\right)$ we choose, so (again somewhat sloppily) we can say that $f$ is almost $\mathcal{A}_{0^{0}}$-measurable if and only if $f=\mathrm{E}\left(f \mid \mathcal{A}_{0}\right)$ almost everywhere.

\subsection{Push-Forward and Quotients}

Let $(\Omega, \mathcal{A}, \pi)$ and $\left(\Omega^{\prime}, \mathcal{A}^{\prime}, \pi^{\prime}\right)$ be probability spaces and let $\phi: \Omega \rightarrow \Omega^{\prime}$ be a measure preserving map. We have described how to "pull back" a graphon on $\left(\Omega^{\prime}, \mathcal{A}^{\prime}, \pi^{\prime}\right)$ to a (weakly isomorphic) graphon on $(\Omega, \mathcal{A}, \pi)$. It is also possible to "push-forward" a graphon $H=(\Omega, \mathcal{A}, \pi, W)$ to a graphon $\left(\Omega^{\prime}, \mathcal{A}^{\prime}, \pi^{\prime}, W_{\phi}\right)$. This is defined by the requirement that

$$
\int_{A_{1}^{\prime} \times A_{2}^{\prime}} W_{\phi}\left(x^{\prime}, y^{\prime}\right) d \pi^{\prime}\left(x^{\prime}\right) d \pi^{\prime}\left(y^{\prime}\right)=\int_{\phi^{-1}\left(A_{1}^{\prime}\right) \times \phi^{-1}\left(A_{2}^{\prime}\right)} W(x, y) d \pi(x) d \pi(y)
$$

for all $A_{1}^{\prime}, A_{2}^{\prime} \in \mathcal{A}^{\prime}$. The next lemma states that the "push-forward" $W_{\phi}$ is well defined, and that $\left(W_{\phi}\right)^{\phi}$ is a certain conditional expectation of $W$.

Lemma 3.1 Let $(\Omega, \mathcal{A}, \pi)$ and $\left(\Omega^{\prime}, \mathcal{A}^{\prime}, \pi^{\prime}\right)$ be probability spaces, let $\phi: \Omega \rightarrow$ $\Omega^{\prime}$ be a measure preserving map, and let $W$ be a graphon on $(\Omega, \mathcal{A}, \pi)$.

(i) There exists a bounded, symmetric function $W_{\phi}: \Omega^{\prime} \times \Omega^{\prime} \rightarrow \mathbb{R}$ that is $\mathcal{A}^{\prime} \times \mathcal{A}^{\prime}$ measurable and satisfies (6) . It is unique up to changes on a set of measure zero in $\Omega^{\prime} \times \Omega^{\prime}$.

(ii) Let $\mathcal{A}_{\phi}=\phi^{-1}\left(\mathcal{A}^{\prime}\right)$. Then $\left(W_{\phi}\right)^{\phi}=\mathrm{E}\left(W \mid \mathcal{A}_{\phi} \times \mathcal{A}_{\phi}\right)$ almost everywhere.

(iii) If $\phi$ is an embedding of $(\Omega, \mathcal{A}, \pi)$ into $\left(\Omega^{\prime}, \mathcal{A}^{\prime}, \pi^{\prime}\right)$, then $\left(W_{\phi}\right)^{\phi}=W$ almost everywhere.

Proof. (i) By linearity, it is easy to see that we can restrict ourselves to the case where $W$ takes values in $[0,1]$. Define a measure $\mu$ on $\mathcal{A}^{\prime} \times \mathcal{A}^{\prime}$ by

$$
\mu\left(A_{1}^{\prime} \times A_{2}^{\prime}\right)=\int_{\phi^{-1}\left(A_{1}^{\prime}\right) \times \phi^{-1}\left(A_{2}^{\prime}\right)} W(x, y) d \pi(x) d \pi(y)
$$


for $A_{1}^{\prime}, A_{2}^{\prime} \in \mathcal{A}$. With this definition, we have that

$$
0 \leq \mu\left(A_{1}^{\prime} \times A_{2}^{\prime}\right) \leq \pi\left(\phi^{-1}\left(A_{1}^{\prime}\right)\right) \pi\left(\phi^{-1}\left(A_{2}^{\prime}\right)\right)=\left(\pi^{\prime} \times \pi^{\prime}\right)\left(A_{1}^{\prime} \times A_{2}^{\prime}\right),
$$

implying in particular that $\mu$ is absolutely continuous with respect to $\pi^{\prime} \times \pi^{\prime}$. Hence the Radon-Nikodym derivative,

$$
W_{\phi}=\frac{d \mu}{d\left(\pi^{\prime} \times \pi^{\prime}\right)},
$$

is well defined. Using the above bound once more, together with the fact that $\mu\left(A_{1} \times A_{2}\right)=\mu\left(A_{2} \times A_{1}\right)$, we furthermore have that

$$
0 \leq W_{\phi}(x, y) \leq 1 \quad \text { and } \quad W_{\phi}(x, y)=W_{\phi}(y, x)
$$

almost everywhere. Changing $W_{\phi}$ on a set of measure zero, we may assume that these relations hold everywhere. To define $W_{\phi}$ for a general bounded function $W$, we use linearity.

(ii) Let $A_{1}, A_{2} \in \mathcal{A}_{\phi}$, i.e., let $A_{1}=\phi^{-1}\left(A_{1}^{\prime}\right)$ and $A_{2}=\phi^{-1}\left(A_{2}^{\prime}\right)$ for some $A_{1}^{\prime}, A_{2}^{\prime} \in \mathcal{A}^{\prime}$. By the definition of $W_{\phi}$, the fact that $\phi$ is measure preserving, and the definition of $\left(W_{\phi}\right)^{\phi}$, we have that

$$
\begin{aligned}
\int_{A_{1} \times A_{2}} W(x, y) d \pi(x) d \pi(y) & =\int_{A_{1}^{\prime} \times A_{2}^{\prime}} W_{\phi}\left(x^{\prime}, y^{\prime}\right) d \pi^{\prime}\left(x^{\prime}\right) d \pi^{\prime}\left(y^{\prime}\right) \\
& =\int_{A_{1} \times A_{2}} W_{\phi}(\phi(x), \phi(y)) d \pi(x) d \pi(y) \\
& =\int_{A_{1} \times A_{2}}\left(W_{\phi}\right)^{\phi}(x, y) d \pi(x) d \pi(y) .
\end{aligned}
$$

This implies that $\left(W_{\phi}\right)^{\phi}=\mathrm{E}\left(W \mid \mathcal{A}_{\phi} \times \mathcal{A}_{\phi}\right)$ almost everywhere.

(iii) Since $\phi$ is an isomorphism between $(\Omega, \mathcal{A}, \pi)$ and a subspace of $\left(\Omega^{\prime}, \mathcal{A}^{\prime}, \pi^{\prime}\right)$, we know that given any $A \in \mathcal{A}$, we can find an $A^{\prime} \in \mathcal{A}^{\prime}$ such that $\phi(A)=A^{\prime} \cap \phi(\Omega)$. But then $\phi^{-1}\left(A^{\prime}\right)=\phi^{-1}(\phi(A))=A$, proving that $A \in \mathcal{A}_{\phi}$. Thus $\mathcal{A}_{\phi}=\mathcal{A}$, which implies that $\left(W_{\phi}\right)^{\phi}=W$ almost everywhere. 
We can use the "push-forward" construction to define quotients of graphons. Let $H=(\Omega, \mathcal{A}, \pi, W)$ be a graphon, let $\mathcal{P}$ be an arbitrary partition of $\Omega$ into disjoint sets, and for $x \in \Omega$, let $[x]$ denote the class in $\mathcal{P}$ that contains the point $x$. We then define a graphon $H / \mathcal{P}=(\Omega / \mathcal{P}, \mathcal{A} / \mathcal{P}, \pi / \mathcal{P}, W / \mathcal{P})$ and a measure preserving map $\phi: \Omega \rightarrow \Omega / \mathcal{P}$ as follows: the points in $\Omega / \mathcal{P}$ are the classes of the partition $\mathcal{P}, \phi$ is the map $\phi: x \mapsto[x], \mathcal{A} / \mathcal{P}$ is the $\sigma$-algebra consisting of the sets $A^{\prime} \subset \Omega / \mathcal{P}$ such that $\phi^{-1}\left(A^{\prime}\right) \in \mathcal{A}$, and $(\pi / \mathcal{P})\left(A^{\prime}\right):=\pi\left(\phi^{-1}\left(A^{\prime}\right)\right)$. Then $\phi$ is measure preserving, and the function $W / \mathcal{P}=W_{\phi}$ is defined by (5).

\subsection{Reductions}

Now we are able to state the theorem that allows us to reduce every graphon to a twin-free Lebesguian graphon.

Theorem 3.2 (i) Let $H=(\Omega, \mathcal{A}, \pi, W)$ be a graphon. Then one can change the value of $W$ on a set of $\pi \times \pi$-measure 0 to get a strong graphon.

(ii) Let $H=(\Omega, \mathcal{A}, \pi, W)$ be a graphon. Then there exists a countably generated $\sigma$-algebra $\mathcal{A}_{0} \subset \mathcal{A}$ such that $W$ is $\left(\mathcal{A}_{0} \times \mathcal{A}_{0}\right)$-measurable.

(iii) Let $H=(\Omega, \mathcal{A}, \pi, W)$ be a graphon. Then the graphon $H / \mathcal{P}[\mathcal{A}]$ is separating. If $H$ is countably generated, then so is $H / \mathcal{P}[\mathcal{A}]$.

(iv) Let $H=(\Omega, \mathcal{A}, \pi, W)$ be a separating graphon on a probability space with a countable basis. Then the completion of $H$ can be embedded into a Lebesguian graphon.

(v) Let $H=(\Omega, \mathcal{A}, \pi, W)$ be a graphon, and let $\mathcal{P}$ be the partition into the twin-classes of $H$. Then $H / \mathcal{P}$ is almost twin-free. If $H$ is Lebesguian, then $H / \mathcal{P}$ is Lebesguian as well. Furthermore, the projection $H \rightarrow H / \mathcal{P}$ is a weak isomorphism.

Corollary 3.3 Every graphon has a weak isomorphism into a strong Lebesguian graphon. 
The proof of this theorem (which is not hard, but technical) will be given in the rest of this section.

\subsubsection{Making a graphon strong}

Let $H=(\Omega, \mathcal{A}, \pi, W)$ be a graphon, and let $W^{\prime}=\mathrm{E}(W \mid \mathcal{A} \times \mathcal{A})$. Then $W^{\prime}$ is $\mathcal{A} \times \mathcal{A}$-measurable, and changing $W^{\prime}$ on a set of measure 0 , we may assume that $W^{\prime}$ is symmetric and bounded. Moreover, $\int_{A \times A^{\prime}}\left(W^{\prime}-W\right)=0$ for all $A, A^{\prime} \in \mathcal{A}$, which implies that $\int_{S}\left(W^{\prime}-W\right)=0$ for all sets $S$ in the completion of $\mathcal{A} \times \mathcal{A}$, so $W=W^{\prime}$ almost everywhere. These observations prove part (i) of the Theorem.

\subsubsection{Countable generation}

We prove a simple lemma, which implies Theorem 3.2 (ii), and will also be used at several other places (Sections 4.1 and 5.2).

Lemma 3.4 Let $(\Omega, \mathcal{A})$ and $\left(\Omega^{\prime}, \mathcal{A}^{\prime}\right)$ be measurable spaces, and let $W$ : $\Omega \times \Omega^{\prime} \rightarrow \mathbb{R}$ be a bounded, $\left(\mathcal{A} \times \mathcal{A}^{\prime}\right)$-measurable function. Then there exist countably generated $\sigma$-algebras $\mathcal{A}_{0} \subset \mathcal{A}$ and $\mathcal{A}_{0}^{\prime} \subset \mathcal{A}^{\prime}$ such that $W$ is $\left(\mathcal{A}_{0} \times \mathcal{A}_{0}^{\prime}\right)$-measurable.

Proof. Let $\mathcal{C}$ be the set of bounded, $\left(\mathcal{A} \times \mathcal{A}^{\prime}\right)$-measurable functions $W$ for which the statement of the lemma is true. The set $\mathcal{C}$ is clearly a vector space that contains the constant function 1 as well as the indicator functions of all rectangles $A \times B$ with $A \in \mathcal{A}$ and $B \in \mathcal{A}^{\prime}$. If is further not hard to show that if $\left(W_{k}\right)$ is a sequence of non-negative functions in $\mathcal{C}$ and $W_{k} \uparrow W$ for a bounded function $W$, then the limiting function $W$ is in $\mathcal{C}$ as well. By the monotone class theorem (see, e.g., Theorem 3.14 in [14]), we conclude that $\mathcal{C}$ contains all bounded functions which are measurable with respect to the $\sigma$-algebra generated by the rectangles $A \times B$, i.e., the $\sigma$-algebra $\mathcal{A} \times \mathcal{A}^{\prime}$. 


\subsubsection{Merging inseparable elements}

If we identify elements in the same class of the partition $\mathcal{P}[\mathcal{A}]$, we get a $\sigma$-algebra which is isomorphic under the obvious map. This implies (iii) of Theorem 3.2 .

\subsubsection{Lebesgue property}

Consider a separating graphon $H=(\Omega, \mathcal{A}, \pi, W)$, and assume that $\mathcal{A}$ is generated by the countable set $\mathcal{S}$. Then $\mathcal{S}$ is a basis for the completion of $(\Omega, \mathcal{A}, \pi)$. We invoke the fact (see e.g. [13], Section 2.2) that any separating complete probability space with a countable basis can be embedded into a Lebesgue space. Thus there exists an embedding $\psi$ of the completion of $(\Omega, \mathcal{A}, \pi)$ into a Lebesgue space $\left(\Omega^{\prime}, \mathcal{L}^{\prime}, \lambda^{\prime}\right)$. Let $W^{\prime}$ be the push-forward of $W, W^{\prime}=W_{\psi}$. By Lemma 3.1, we have that $\left(W^{\prime}\right)^{\psi}=W$ almost everywhere, which shows that $\psi$ is an embedding of the completion of $H$ into $\left(\Omega^{\prime}, \mathcal{L}^{\prime}, \lambda^{\prime}, W^{\prime}\right)$. This proves part (iv) of Theorem 3.2 .

\subsubsection{Partitions into Twin-Classes}

We prove (v) in Theorem 3.2. We may assume that $\mathcal{A}$ is countably generated. Indeed, by Lemma 3.4, we can replace $\mathcal{A}$ by a countably generated $\sigma$-algebra $\mathcal{A}_{0}$. This does not change the relation of being twins: Two points $x, x^{\prime} \in \Omega$ are twins if and only if the set $A_{x, x^{\prime}}=\left\{y \in \Omega: W(x, y)=W\left(x, y^{\prime}\right)\right\}$ has

measure 1 . Since $W$ is measurable with respect to $\mathcal{A}_{0} \times \mathcal{A}_{0}$, the set $A_{x, x^{\prime}}$ lies in $\mathcal{A}_{0} \subset \mathcal{A}$, implying that $x$ and $x^{\prime}$ are twins with respect to $H$ if and only if they are twins with respect to $H_{0}$.

Let $\mathcal{A}_{\mathcal{P}}$ consists of those sets in $\mathcal{A}$ that do not separate any pair of twin points. Clearly $\mathcal{A}_{\mathcal{P}}$ is a $\sigma$-algebra.

Claim $1 W$ is almost $\mathcal{A}_{\mathcal{P}} \times \mathcal{A}_{\mathcal{P}}$-measurable.

Let $\widetilde{W}=\mathrm{E}\left(W \mid \mathcal{A}_{\mathcal{P}} \times \mathcal{A}_{\mathcal{P}}\right)$. We want to prove that

$$
\int_{A \times B} W(x, y) d \pi(x) d \pi(y)=\int_{A \times B} \widetilde{W}(x, y) d \pi(x) d \pi(y)
$$


for all $A, B \in \mathcal{A}$. Define the functions

$g_{A}=\mathrm{E}\left(1_{A} \mid \mathcal{A}_{\mathcal{P}}\right), \quad U_{A}(y)=\int_{A} W(x, y) d \pi(x), \quad V_{A}(x)=\int W(x, y) g_{A}(y) d \pi(y)$.

Since $U_{A}(y)=U_{A}(z)$ if $y, z$ are twins, the function $U_{A}$ is $\mathcal{A}_{\mathcal{P}}$-measurable, similarly for $V_{A}$, and obviously for $g_{A}$. Repeatedly using the fact that $\int f g=$ $\int f \mathrm{E}\left(g \mid \mathcal{A}_{0}\right)$ if $f$ is $\mathcal{A}_{0}$-measurable, this implies

$$
\begin{aligned}
\int_{A \times B} W(x, y) d \pi(x) d \pi(y) & =\int 1_{B}(y) U_{A}(y) d \pi(y)=\int g_{B}(y) U_{A}(y) d \pi(y) \\
& =\int V_{B}(x) 1_{A}(x) d \pi(x)=\int V_{B}(x) g_{A}(x) d \pi(x) \\
& =\int W(x, y) g_{A}(x) g_{B}(y) d \pi(x) d \pi(y) \\
& =\int \widetilde{W}(x, y) g_{A}(x) g_{B}(y) d \pi(x) d \pi(y) \\
& =\int_{A \times B} \tilde{W}(x, y) d \pi(x) d \pi(y) .
\end{aligned}
$$

(where the last equality follows since $\widetilde{W}$ is $\mathcal{A}_{\mathcal{P}} \times \mathcal{A}_{\mathcal{P}}$-measurable). This implies (8) and completes the proof of Claim 1 .

Let $\widetilde{W}=\mathrm{E}\left(W \mid \mathcal{A}_{\mathcal{P}} \times \mathcal{A}_{\mathcal{P}}\right)$ as before, then $H_{\mathcal{P}}=\left(\Omega, \mathcal{A}_{\mathcal{P}}, \pi, \widetilde{W}\right)$ is a graphon, which is clearly weakly isomorphic to $(\Omega, \mathcal{A}, \pi, W)$. Let $N$ be the set of points $x \in \Omega$ for which $\{y \in \Omega: \widetilde{W}(x, y) \neq W(x, y)\}$ has positive measure. Then clearly $N$ is a null set, and two points $x, x^{\prime} \in \Omega \backslash N$ are twins in $H$ if and only if they are twins in $H_{\mathcal{P}}$. The graphon $H / \mathcal{P}$ is obtained from $H_{\mathcal{P}}$ by identifying indistinguishable elements, which implies that $H / \mathcal{P}$ is twin-free.

To prove that $H / \mathcal{P}$ is Lebesguian if $H$ is Lebesguian, we invoke the fact (established in Section 3.2 of [13]) that $(\Omega / \mathcal{P}, \mathcal{A} / \mathcal{P}, \pi / \mathcal{P})$ is a Lebesgue space provided $(\Omega, \mathcal{A}, \pi)$ is a Lebesgue space and there exists a countable set $\mathcal{S} \subseteq \mathcal{A}$ that separates two points if and only they are in different partition classes.

To construct such a set $\mathcal{S}$, let $\mathcal{T}$ be a countable set generating $\mathcal{A}$, closed 
under finite intersections. For $A \in \mathcal{A}$ and $x \in \Omega$, let

$$
\mu_{x}(A)=\int_{A} W(x, y) d \pi(y)
$$

Since $W$ is a bounded $\mathcal{A} \times \mathcal{A}$-measurable function, the function $A \mapsto \mu_{x}(A)$ is a finite measure for all $x \in \Omega$, while the function $x \mapsto \mu_{x}(A)$ is a $\mathcal{A}$ measurable function on $\Omega$ for all $A \in \mathcal{A}$.

By definition, $x, x^{\prime} \in \Omega$ are twins iff the set $\left\{y \in \Omega: W(x, y)=W\left(x, y^{\prime}\right)\right\}$ has measure zero. This is equivalent to the condition that $\mu_{x}(A)=\mu_{x^{\prime}}(A)$ for all $A \in \mathcal{A}$. Since the measure $\mu_{x}(\cdot)$ on $\mathcal{A}$ is uniquely determined by the sets in $\mathcal{T}$, we have that $x$ and $x^{\prime}$ are twins if and only if $\mu_{x}(T)=\mu_{x^{\prime}}(T)$ for all $T \in \mathcal{T}$.

For every $T \in \mathcal{T}$ and rational number $r$, consider the sets $S_{T, r}=\{x \in$ $\left.\Omega: \mu_{x}(T) \geq r\right\}$. There is a countable number of these. Furthermore, if $x$ and $x^{\prime}$ are twins, then they belong to exactly the same sets $S_{T, r}$; if they are not twins, then there is a $T \in \mathcal{T}$ such that $\mu_{x}(T) \neq \mu_{x^{\prime}}(T)$, and for any rational number between $\mu_{x}(T)$ and $\mu_{x^{\prime}}(T)$, the set $S_{T, r}$ separates $x$ and $x^{\prime}$.

This completes the proof of Theorem 3.2 .

\subsection{Isomorphism and Weak Isomorphism}

We conclude this section with relating isomorphism and weak isomorphism.

Lemma 3.5 Let $H_{i}=\left(\Omega_{i}, \mathcal{A}_{i}, \pi_{i}, W_{i}\right)$ be graphons with the Lebesgue property $(i=1,2)$, and let $\phi: \Omega_{1} \rightarrow \Omega_{2}$ be measure-preserving. If $H_{1}$ is almost twinfree, and $W_{1}=W_{2}^{\phi}$ almost everywhere, then $\phi$ is an isomorphism mod 0 , so in particular $H_{1} \cong H_{2}$.

Proof. Let

$$
\Omega_{1}^{\prime}=\left\{x \in \Omega_{1}: W_{2}(\phi(x), \phi(y))=W_{1}(x, y) \text { for almost all } y\right\}
$$

and let $N_{1}=\Omega_{1} \backslash \Omega_{1}^{\prime}$. Then $\pi_{1}\left(N_{1}\right)=0$ by Fubini and our assumption that $W_{1}=W_{2}^{\phi}$ almost everywhere. 
Let $N_{1}^{\prime}$ be a nullset such that all twin-classes of $H_{1}$ have at most one point in $\Omega_{1} \backslash N_{1}^{\prime}$, and let $\phi^{\prime}$ to be the restriction of $\phi$ to $\Omega_{1}^{\prime} \backslash N_{1}^{\prime}$. Then $\phi^{\prime}$ is injective: indeed, if $x_{1}, x_{2} \in \Omega_{1}^{\prime} \backslash N_{1}^{\prime}$ and $\phi\left(x_{1}\right)=\phi\left(x_{2}\right)$, then $W_{1}\left(x_{1}, y\right)=W_{2}\left(\phi\left(x_{1}\right), \phi(y)\right)=W_{2}\left(\phi\left(x_{2}\right), \phi(y)\right)=W_{1}\left(x_{2}, y\right)$ for almost all $y$ by the definition of $\Omega_{1}^{\prime}$, hence $x_{1}$ and $x_{2}$ are twins, a contradiction. As shown in [13], Section 2.5, an injective measure preserving map between Lebesgue spaces has a measurable inverse defined almost everywhere. This implies that $\phi^{\prime}: \Omega_{1}^{\prime} \backslash N_{1}^{\prime} \rightarrow \Omega_{2}$ is an isomorphism mod 0 , which shows that $\phi$ is an isomorphism mod 0 as well.

Corollary 3.6 If two twin-free graphons with the Lebesgue property are weakly isomorphic, then they are isomorphic.

\section{Canonical Ensembles}

We could try to construct a "canonical form" of a graphon by assigning "tags" to the points in $\Omega$. For example, we could tag a point $x$ with its marginal $d(x)=\int W(x, y) d \pi(y)$, or by the sequence of marginals of higher powers of $W$. This, however, would not work: for example, there could be a transitive group of measure-preserving permutations of $\Omega$ leaving $W$ invariant, and then all points would still have the same tag.

To break the symmetry, we select an infinite sequence $\alpha=\left(a_{1}, a_{2}, \ldots\right)$ of points in $\Omega$, which we call anchor points. Now we can tag each point $x \in \Omega$ with the sequence

$$
\Phi_{\alpha}(x)=\left(W\left(x, a_{1}\right), W\left(x, a_{2}\right), \ldots\right) \in[0,1]^{\mathbb{N}}
$$

(where we assume that $0 \leq W \leq 1$ ) The map $x \mapsto \Phi_{\alpha}(x)$ defines a measurable map from $\Omega$ into $[0,1]^{\mathbb{N}}$ (with respect to the standard Borel $\sigma$-algebra $\mathcal{L}$ on $\left.[0,1]^{\mathbb{N}}\right)$, which in turn defines a measure $\lambda_{\alpha}$ on the sets $S \in \mathcal{L}$ by

$$
\lambda_{\alpha}(S)=\pi\left(\Phi_{\alpha}^{-1}(S)\right),
$$


and a graphon $W_{\Phi_{\alpha}}$ on $\left([0,1]^{\mathbb{N}}, \mathcal{L}, \lambda_{\alpha}\right)$ by (5). We denote the completion of $\left([0,1]^{\mathbb{N}}, \mathcal{L}, \lambda_{\alpha}, W_{\Phi_{\alpha}}\right)$ by $H_{\alpha}$.

We will show that if $\alpha_{1}, \alpha_{2}, \ldots$ are taken i.i.d. at random with distribution $\pi$ then with probability one, then $H_{\alpha}$ is isomorphic mod 0 to the original graphon $H$ (see Section 4.2 for details). So using an infinite sequence of independent random points as anchor points, the tags of the points contain all information about the points.

These tags are almost canonical, except for the choice of the sequence $\alpha$. So instead of a canonical form, we get a "canonical ensemble", a probability distribution $\left(H_{\alpha}\right)$ of graphons such that $H \cong H_{\alpha}$ for almost all $\alpha$, and two graphons are isomorphic if and only if their ensembles can be coupled so that corresponding graphons are isomorphic.

To prove Theorem 2.1 (i), we will therefore have to show that if $H$ and $H^{\prime}$ satisfy (4), then we can "couple" the choice of anchor points $\alpha$ in $H$ and $\beta$ in $H^{\prime}$ so that $H_{\alpha} \cong H_{\beta}^{\prime}$, thus yielding an isomorphism of $H$ and $H^{\prime}$. This second step in the proof will be carried out in Section 5.3.

\subsection{Measure theoretic preparation}

The next technical lemma will be important in the construction of "canonical ensembles".

Lemma 4.1 Let $(\Omega, \mathcal{A}, \pi)$ and $\left(\Omega^{\prime}, \mathcal{A}^{\prime}, \pi^{\prime}\right)$ be probability spaces, and let $W$ : $\Omega \times \Omega^{\prime} \rightarrow \mathbb{R}$ be a bounded $\mathcal{A} \times \mathcal{A}^{\prime}$-measurable function. Let $Y_{1}, Y_{2}, \ldots$ be independent random points from $\Omega^{\prime}$. Let $\mathcal{A}_{0} \subseteq \mathcal{A}$ be the (random) $\sigma$-algebra generated by the functions $W\left(\cdot, Y_{k}\right)$. Then with probability $1, W$ is almost $\mathcal{A}_{0} \times \mathcal{A}^{\prime}$-measurable.

Proof. By Lemma 3.4, we may assume that $\mathcal{A}$ and $\mathcal{A}^{\prime}$ are countably generated. Let $\mathcal{A}_{1}^{\prime} \subset \mathcal{A}_{2}^{\prime} \subset \ldots$ and $\mathcal{A}_{1}^{\prime} \subset \mathcal{A}_{2}^{\prime} \subset \ldots$ be a sequence of finite $\sigma$-algebras with $\sigma\left(\cup_{n} \mathcal{A}_{n}\right)=\mathcal{A}$ and $\sigma\left(\cup_{n} \mathcal{A}_{n}^{\prime}\right)=\mathcal{A}^{\prime}$, and let $P_{n}^{\prime}$ denote the 
partition of $\Omega^{\prime}$ into the atoms of $\mathcal{A}_{n}^{\prime}$. For $y \in S \in P_{n}^{\prime}$ with $\pi^{\prime}(S)>0$, define

$$
U_{n, m}(x, y)=\frac{1}{m \pi(S)} \sum_{\substack{j \leq m \\ Y_{j} \in S}} W\left(x, Y_{j}\right)
$$

We define $U_{n, m}(x, y)=0$ if $y \in S \in P_{n}^{\prime}$ with $\pi^{\prime}(S)=0$.

First we prove that for every $n \geq 1$, every $A \in \mathcal{A}$ and $A^{\prime} \in \mathcal{A}_{n}^{\prime}$, we have with probability 1

$$
\int_{A \times A^{\prime}} U_{n, m} d \pi d \pi^{\prime} \longrightarrow \int_{A \times A^{\prime}} W d \pi d \pi^{\prime} \quad(m \rightarrow \infty) .
$$

It suffices to prove this in the case when $A^{\prime}=S \in P_{n}^{\prime}$ and $\pi^{\prime}(S)>0$. Then for every $y_{0} \in A^{\prime}$, we have

$$
\int_{A} U_{n, m}\left(x, y_{0}\right) d \pi(x)=\frac{1}{m \pi(S)} \sum_{\substack{j \leq m \\ Y_{j} \in S}} \int_{A} W\left(x, Y_{j}\right) d \pi(x) .
$$

hence by the Law of Large Numbers,

$$
\int_{A} U_{n, m}\left(x, y_{0}\right) d \pi(x) \longrightarrow \frac{1}{\pi(S)} \int_{A \times S} W d \pi d \pi^{\prime} \quad(m \rightarrow \infty) .
$$

Since both sides are independent of $y_{0} \in S$, integrating over $y_{0} \in S$ equation (11) follows.

The number of choices of $n, A \in \cup_{k} \mathcal{A}_{k}$ and $A^{\prime} \in \mathcal{A}_{n}^{\prime}$ is countable, and hence it follows that with probability 1, (11) holds for all $n \geq 1$, every $A \in \cup_{k} \mathcal{A}_{k}$ and $A^{\prime} \in \mathcal{A}_{n}^{\prime}$. Since $\cup_{k} \mathcal{A}_{k}$ is dense in $\mathcal{A}$, this implies that (11) holds for all $n \geq 1$, every $A \in \mathcal{A}$ and $A^{\prime} \in \mathcal{A}_{n}^{\prime}$.

From now on, we suppose that the choice of the $Y_{i}$ is such that this holds.

For a fixed $n$, the indices $m$ have a subsequence $m_{1}<m_{2}<\ldots$ such that $U_{n, m_{j}}$ converges to some function $U_{n}$ in the weak-*-topology of $L_{\infty}\left(\mathcal{A}_{0} \times \mathcal{A}_{n}^{\prime}\right)$. Hence by (11),

$$
\int_{A \times A^{\prime}} U_{n} d \pi d \pi^{\prime}=\lim _{j \rightarrow \infty} \int_{A \times A^{\prime}} U_{n, m_{j}} d \pi d \pi^{\prime}=\int_{A \times A^{\prime}} W d \pi d \pi^{\prime}
$$


for all $n \geq 1$, every $A \in \mathcal{A}$ and $A^{\prime} \in \mathcal{A}_{n}^{\prime}$. Thus $U_{n}$ is a representative of $\mathrm{E}\left(W \mid \mathcal{A} \times \mathcal{A}_{n}^{\prime}\right)$. Since $U_{n}$ is $\mathcal{A}_{0} \times \mathcal{A}_{n}^{\prime}$ measurable, it is also a representative of $\mathrm{E}\left(W \mid \mathcal{A}_{0} \times \mathcal{A}_{n}^{\prime}\right)$. This shows that for every $n \geq 1$ we have

$$
\mathrm{E}\left(W \mid \mathcal{A} \times \mathcal{A}_{n}^{\prime}\right)=\mathrm{E}\left(W \mid \mathcal{A}_{0} \times \mathcal{A}_{n}^{\prime}\right)
$$

almost everywhere.

By Levy's Upward Theorem, the left hand side of (12) tends to $\mathrm{E}(W$ $\left.\mathcal{A} \times \mathcal{A}^{\prime}\right)=W$ almost everywhere. The right hand side of (12) tends to $\mathrm{E}(W \mid$ $\left.\mathcal{A}_{0} \times \mathcal{A}^{\prime}\right)$ almost everywhere, so $W=\mathrm{E}\left(W \mid \mathcal{A}_{0} \times \mathcal{A}^{\prime}\right)$ almost everywhere, which proves the Lemma.

We formulate a couple of corollaries, the first of which is immediate:

Corollary 4.2 Let $(\Omega, \mathcal{A}, \pi)$ and $\left(\Omega^{\prime}, \mathcal{A}^{\prime}, \pi^{\prime}\right)$ be probability spaces, let $W: \Omega \times \Omega^{\prime} \rightarrow \mathbb{R}$ be a bounded function that is measurable with respect to $\mathcal{A} \times \mathcal{A}^{\prime}$, and let $\mathcal{A}_{0} \subset \mathcal{A}$ be a sub- $\sigma$-algebra. If $W(\cdot, y)$ is $\mathcal{A}_{0}$-measurable for almost all $x \in \Omega$, then $W$ is almost $\mathcal{A}_{0} \times \mathcal{A}^{\prime}$-measurable.

Corollary 4.3 Let $(\Omega, \mathcal{A}, \pi, W)$ be a graphon, and let $X_{1}, X_{2}, \ldots$ be independent random points from $\Omega$. Let $\mathcal{A}_{0} \subseteq \mathcal{A}$ be the (random) $\sigma$-algebra generated by the functions $W\left(\cdot, X_{k}\right)$. Then with probability $1, W$ is almost $\mathcal{A}_{0} \times \mathcal{A}_{0}$-measurable.

Proof. Let $\mathcal{A}_{1}$ denote the $\sigma$-algebras generated by the functions $W\left(\cdot, X_{2 k}\right)$. Clearly $\mathcal{A}_{1} \subseteq \mathcal{A}_{0}$. By Lemma 4.1, $W$ is almost $\mathcal{A}_{1} \times \mathcal{A}$ measurable with probability 1 , so we can change it on a set of measure 0 to get an $\mathcal{A}_{1} \times \mathcal{A}$ measurable function $W^{\prime}$. Let $\mathcal{A}_{2}^{\prime}$ be the $\sigma$-algebras generated by the functions $W^{\prime}\left(X_{2 k+1}, \cdot\right)$. Applying the lemma again, we get that $W^{\prime}$ is almost $\mathcal{A}_{1} \times$ $\mathcal{A}_{2}^{\prime}$ measurable. With probability 1 , each function $W\left(X_{2 k+1}, \cdot\right)$ differs from $W^{\prime}\left(X_{2 k+1}, \cdot\right)$ on a set of measure 0 only (since the $X_{2 k+1}$ are independent of $\left.\mathcal{A}_{1}\right)$, and so $\mathcal{A}_{2}^{\prime} \subseteq \sigma\left(\mathcal{A}_{0}\right)$. So $W^{\prime}$ is $\mathcal{A}_{0} \times \sigma\left(\mathcal{A}_{0}\right)$ measurable, which implies that $W^{\prime}$, and hence $W$, are almost $\mathcal{A}_{0} \times \mathcal{A}_{0}$ measurable. 


\subsection{Anchor Sequences}

Let us consider the $\sigma$-algebra $\mathcal{L}$ on $[0,1]^{\mathbb{N}}$ generated by the sets $A_{1} \times A_{2} \times \ldots$, where each $A_{i}$ is a Borel subset of $[0,1]$ and only a finite number of factors $A_{i}$ are different from $[0,1]$. Fix a graphon $H=(\Omega, \mathcal{A}, \pi, W)$ with $0 \leq$ $W \leq 1$. For every $\alpha \in \Omega^{\mathbb{N}}$, the map $\Phi_{\alpha}: \Omega \rightarrow[0,1]^{\mathbb{N}}$ defined by (91) is measurable, and (10) defines a probability measure on $\mathcal{L}$ with respect to which $\Phi_{\alpha}$ is measure preserving. Thus (6) leads to a symmetric, $\mathcal{L} \times \mathcal{L}$ measurable function $W_{\Phi_{\alpha}}:[0,1]^{\mathbb{N}} \times[0,1]^{\mathbb{N}} \rightarrow[0,1]$ which we denote by $W_{\alpha}$. We say that $\alpha \in \Omega^{\mathbb{N}}$ is regular if $W=W_{\alpha}^{\Phi_{\alpha}}$ almost everywhere.

Lemma 4.4 Almost all $\alpha \in \Omega^{\mathbb{N}}$ are regular.

Proof. Let $\mathcal{A}_{\alpha}$ denote the $\sigma$-algebra of subsets of $\Omega$ of the form $\Phi_{\alpha}^{-1}(A)$, where $A \in \mathcal{L}$. Note that $\mathcal{A}_{\alpha} \subseteq \mathcal{A}$ by the fact that $\Phi_{\alpha}$ is measurable. Further, almost by definition, $\mathcal{A}_{\alpha}$ is the smallest sub- $\sigma$-algebra of $\mathcal{A}$ such that all the functions $W\left(\cdot, \alpha_{i}\right)$ are measurable. As a consequence, we may apply Lemma 4.3 to conclude that for almost all $\alpha, W$ is almost $\mathcal{A}_{\alpha} \times \mathcal{A}_{\alpha}$ )-measurable, which by Lemma 3.1 gives that $W=W_{\alpha}^{\Phi_{\alpha}}$ almost everywhere.

Let $\mathcal{L}_{\alpha}$ be the completion of $\mathcal{L}$ with respect to $\lambda_{\alpha}$. Then $\left([0,1]^{\mathbb{N}}, \mathcal{L}_{\alpha}, \lambda_{\alpha}\right)$ is a complete, Polish space and hence Lebesgue, so $H_{\alpha}=\left([0,1]^{\mathbb{N}}, \mathcal{L}_{\alpha}, \lambda_{\alpha}, W_{\alpha}\right)$ defines a Lebesguian graphon.

Lemma 4.5 Let $H$ be a twin free graphon with the Lebesgue property. If $\alpha$ is regular, then $\Phi_{\alpha}$ is an isomorphism $\bmod 0$ and $H_{\alpha} \cong H$.

Proof. By (10), $\Phi_{\alpha}$ is a measure preserving map from $(\Omega, \mathcal{A}, \pi)$ into $\left([0,1]^{\mathbb{N}}, \mathcal{L}, \lambda_{\alpha}\right)$. Since $(\Omega, \mathcal{A}, \pi)$ is complete, $\Phi_{\alpha}$ is measurable (and measure preserving) from $(\Omega, \mathcal{A}, \pi)$ into $\left([0,1]^{\mathbb{N}}, \mathcal{L}_{\alpha}, \lambda_{\alpha}\right)$ as well. By the definition of a regular $\alpha, H_{\alpha}^{\phi_{\alpha}}=H$ almost everywhere, and by Lemma 3.5, $\Phi_{\alpha}$ is an isomorphism mod 0 . 


\section{Coupling}

\subsection{Partially Labeled Graphs and Marginals}

We recall some notions from [8]. A partially labeled graph is a finite graph in which some of the nodes are labeled by different nonnegative integers. Two partially labeled graphs are isomorphic, if there is a label-preserving isomorphism between them. A k-labeled graph is a partially labeled graph with labels $1, \ldots, k$.

Let $F_{1}$ and $F_{2}$ be two partially labeled graphs. Their product $F_{1} F_{2}$ is defined as follows: we take their disjoint union, and then identify nodes with the same label (retaining the labels, and any multiple edges which this might create). For two unlabeled graphs, $F_{1} F_{2}$ is their disjoint union. Clearly this multiplication is associative and commutative.

Let $H=(\Omega, \mathcal{A}, \pi, W)$ be a graphon, and let $\alpha=\left(a_{0}, a_{1}, \ldots\right)$ be an infinite sequence of points in $\Omega$. Let $F$ be a partially labeled graph with nodes $V(F)=\{1, \ldots, k\}$, where nodes $1, \ldots, r$ are labeled by distinct nonnegative integers $\ell_{1}, \ldots, \ell_{r}$. Let $X_{i}=a_{\ell_{i}}$ for $1 \leq i \leq r$, and let $X_{r+1}, \ldots, X_{k} \in \Omega$ be independent points from the distribution $\pi$. Define

$$
t_{\alpha}(F, H)=\mathrm{E}\left(\prod_{i j \in E(F)} W\left(X_{j}, X_{j}\right)\right) .
$$

Of course, this value only depends on those elements of $\alpha$ whose subscripts occur as labels, and we'll sometimes omit the tail of $\alpha$ if it contains no labels. For example, if $F$ is a 2-labeled triangle, then

$$
\begin{aligned}
t_{a_{1} a_{2}}(F, H) & =t_{\alpha}(F, W)=\mathrm{E}\left(W\left(a_{1}, a_{2}\right) W\left(a_{2}, X\right) W\left(a_{1}, X\right)\right) \\
& =\int_{\Omega} W\left(a_{1}, a_{2}\right) W\left(a_{2}, x\right) W\left(a_{1}, x\right) d \pi(x) .
\end{aligned}
$$

It is easy to see that if $F_{1}$ and $F_{2}$ are two $k$-labeled graphs, then

$$
t\left(F_{1} F_{2}, H\right)=\int_{\Omega^{k}} t_{x_{1} \ldots x_{k}}\left(F_{1}, W\right) t_{x_{1} \ldots x_{k}}\left(F_{2}, W\right) d \pi\left(x_{1}\right) \ldots d \pi\left(x_{k}\right) .
$$




\subsection{Multiple Edges}

Lemma 5.1 Let $H=(\Omega, \mathcal{A}, \pi, W)$ and $H^{\prime}=\left(\Omega^{\prime}, \mathcal{A}^{\prime}, \pi^{\prime}, W^{\prime}\right)$ be two graphons, and assume that $t(F, H)=t\left(F, H^{\prime}\right)$ for every simple graph $F$. Then $t(F, H)=t\left(F, H^{\prime}\right)$ for every multigraph $F$.

Proof. We use induction on the number of parallel edges in F. Suppose that $F$ has two nodes, say $i$ and $j$, connected by more than one edge. Let $F_{k}$ denote the multigraph obtained from $F$ by subdividing one of these edges by $k-1$ new nodes. Let $F^{\prime}$ denote the multigraph obtained by removing one copy of the edge $i j$. So $F_{1}=F$, but for $k>1, F_{k}$ has fewer parallel edges than $F$, and so we may assume that

$$
t\left(F_{k}, H\right)=t\left(F_{k}, H^{\prime}\right)
$$

holds for every $k \geq 2$. We consider all the multigraphs $F_{k}$ and $F^{\prime}$ as 2-labeled graphs, with $i$ and $j$ labeled 1 and 2 .

Since $F_{k}$ can be thought of as the product of $F^{\prime}$ and a path $P_{k+1}$ with $k+1$ nodes (the endpoints labeled), we can write

$$
t(F, H)=\int_{\Omega^{2}} W(x, y) t_{x y}\left(F^{\prime}, H\right) d \pi(x) d \pi(y)
$$

and

$$
t\left(F_{k}, H\right)=\int_{\Omega^{2}} t_{x y}\left(P_{k+1}, H\right) t_{x y}\left(F^{\prime}, H\right) d \pi(x) d \pi(y) .
$$

The first factor inside the integral can be expressed as

$$
t_{x y}\left(P_{k+1}, H\right)=\int_{\Omega^{k}} W\left(x, x_{1}\right) \cdots W\left(x_{k-1} y\right) d \pi\left(x_{1}\right) \ldots d \pi\left(x_{k-1}\right),
$$

which we can recognize as $k$-th power of the kernel $W$ as an integral operator.

At this point, it will be useful to assume that $H$ and $H^{\prime}$ are countably generated graphons (this can be done without loss of generality by Lemma 3.4). As a consequence, $W$ is an integral operator on the separable Hilbert space 
$L_{2}(\Omega, \mathcal{A}, \pi)$, and since $W$ is bounded, this implies that $W$ is Hilbert-Schmidt and thus compact, which in turn implies that $W$ has a spectral representation:

$$
W(x, y) \sim \sum_{n=0}^{\infty} \lambda_{n} \varphi_{n}(x) \varphi_{n}(y) .
$$

It follows that for every $k \geq 2$,

$$
t_{x y}\left(P_{k+1}, H\right)=\sum_{n=0}^{\infty} \lambda_{n}^{k} \varphi_{n}(x) \varphi_{n}(y)
$$

and hence

$$
t\left(F_{k}, H\right)=\sum_{n=0}^{\infty} \lambda_{n}^{k} \int_{\Omega^{2}} \varphi_{n}(x) \varphi_{n}(y) t_{x y}\left(F^{\prime}, H\right) d \pi(x) d \pi(y)
$$

Similarly, let

$$
W^{\prime}(x, y) \sim \sum_{i=0}^{\infty} \mu_{n} \psi_{n}(x) \psi_{n}(y)
$$

be the spectral representation of $W^{\prime}$, then we get that for every $k \geq 2$,

$$
0=t\left(F_{k}, H\right)-t\left(F_{k}, H^{\prime}\right)=\sum_{n=0}^{\infty} a_{n} \lambda_{n}^{k}-b_{n} \mu_{n}^{k}
$$

where

$$
a_{n}=\int_{\Omega^{2}} \varphi_{n}(x) \varphi_{n}(y) t_{x y}\left(F^{\prime}, U\right) d \pi(x) d \pi(y)
$$

and

$$
b_{n}=\int_{\left(\Omega^{\prime}\right)^{2}} \psi_{n}(x) \psi_{n}(y) t_{x y}\left(F^{\prime}, W\right) d \pi(x) d \pi(y)
$$

are independent of $k$. (The integrals exist since $t_{x, y}\left(F^{\prime}, H\right)$ is a bounded function of $x$ and $y$.) It follows that in (14) everything must cancel, in other words, for every value $c$,

$$
\sum\left\{a_{n}: \lambda_{n}=c\right\}=\sum\left\{b_{n}: \mu_{n}=c\right\}
$$


(it is known that the sums on both sides have a finite number of terms, since the multiplicities of the eigenvalues are finite).

Now while (13) may not be true with equality, the "trace" with any other kernel gives an equation; in particular,

$$
t(F, H)=\sum_{n=0}^{\infty} \lambda_{n} \int_{\Omega^{2}} W(x, y) t_{x y}\left(F^{\prime}, H\right) d \pi(x) d \pi(y)=\sum_{n=0}^{\infty} a_{n} \lambda_{n}
$$

and similarly

$$
t\left(F, H^{\prime}\right)=\sum_{n=0}^{\infty} b_{n} \mu_{n}
$$

which shows that $t(F, H)=t\left(F, H^{\prime}\right)$ as claimed.

It will be convenient to assume that $0 \leq W, W^{\prime} \leq 1$. If this does not hold, we can apply a linear transformation to the values of the functions, to get two functions $W_{0}$ and $W_{0}^{\prime}$ with $0 \leq W_{0}, W_{0}^{\prime} \leq 1$. Expanding the product in the definition (3),$t\left(F, W_{0}\right)$ can be written as a linear combination of the values $t\left(F^{\prime}, W\right)$, where $F^{\prime}$ is a subgraph of $F$. Thus $t(F, W)=t\left(F, W^{\prime}\right)$ for every graph $F$ if and only if $t\left(F, W_{0}\right)=t\left(F, W_{0}^{\prime}\right)$ for every graph $F$ (where "graph" could mean either simple graph or multigraph). So (4) holds for $W_{0}$ and $W_{0}^{\prime}$ if and only if it holds for $W$ and $W^{\prime}$. If we prove that this implies $\left(\Omega, \mathcal{A}, \pi, W_{0}\right) \cong\left(\Omega^{\prime}, \mathcal{A}^{\prime}, \pi^{\prime}, W_{0}^{\prime}\right)$, then $H \cong H^{\prime}$ follows trivially.

\subsection{Coupling Anchor Sequences}

Consider two graphons $H=(\Omega, \mathcal{A}, \pi, W)$ and $H^{\prime}=\left(\Omega^{\prime}, \mathcal{A}^{\prime}, \pi^{\prime}, W^{\prime}\right)$ satisfying the conditions in Theorem 2.1 (i) and $0 \leq W, W^{\prime} \leq 1$. Given two "anchor" sequences $\alpha=\left(a_{1}, a_{2}, \ldots\right)$ from $\Omega$ and $\beta=\left(b_{1}, b_{2}, \ldots\right)$ from $\Omega^{\prime}$, let $H_{\alpha}=\left([0,1]^{\mathbb{N}}, \mathcal{L}_{\alpha}, \lambda_{\alpha}, W_{\alpha}\right)$ and $H_{\beta}^{\prime}=\left([0,1]^{\mathbb{N}}, \mathcal{L}_{\beta}^{\prime}, \lambda_{\beta}^{\prime}, W_{\beta}^{\prime}\right)$. We would like to select $\alpha$ and $\beta$ in such a way that $\lambda_{\alpha}=\lambda_{\beta}^{\prime}$ and $W_{\alpha}=W_{\beta}^{\prime}$ almost everywhere. This will complete the proof of the theorem. By Lemma 4.4, we can guarantee that both $\alpha$ and $\beta$ are regular by selecting $a_{1}, a_{2}, \ldots$ as well as $b_{1}, b_{2} \ldots$ independently and uniformly from $\pi$ and $\pi^{\prime}$, respectively; however, the equality of $W_{\alpha}$ and $W_{\beta}^{\prime}$ will only be true if we couple $\alpha$ and $\beta$ carefully. 
The condition on the coupling is described in the following lemma.

Lemma 5.2 Let $H=(\Omega, \mathcal{A}, \pi, W)$ and $H^{\prime}=\left(\Omega^{\prime}, \mathcal{A}^{\prime}, \pi^{\prime}, W^{\prime}\right)$ be two graphons, and let $\alpha=\left(a_{1}, a_{2}, \ldots\right)$ and $\beta=\left(b_{1}, b_{2}, \ldots\right)$ be regular sequences for $H$ and $H^{\prime}$, respectively. Suppose that for every partially labeled multigraph $F$,

$$
t_{\alpha}(F, H)=t_{\beta}\left(F, H^{\prime}\right) .
$$

Then $\lambda_{\alpha}=\lambda_{\beta}^{\prime}$ and $W_{\alpha}=W_{\beta}^{\prime}$ almost everywhere (with respect to $\lambda_{\alpha}=\lambda_{\beta}^{\prime}$ ).

Proof. First, we show that $\lambda_{\alpha}=\lambda_{\beta}^{\prime}$. These probability measures are defined on the $\sigma$-algebra $\mathcal{L}$ as the distribution measures of the random variables $\left.W\left(X, a_{1}\right), W\left(X, a_{2}\right), \ldots\right)$ and $\left.W^{\prime}\left(Y, b_{1}\right), W^{\prime}\left(Y, b_{2}\right), \ldots\right)$, where $X$ and $Y$ are random points from $\pi$ and $\pi^{\prime}$, respectively. By Lemma 6.1 it therefore suffices to prove that these random variables have the same mixed moments.

Let $\left(k_{1}, k_{2}, \ldots\right)$ be a sequence of nonnegative integers, of which only a finite number is nonzero; say $k_{i}=0$ for $i>m$. Then

$$
\mathrm{E}\left(\prod_{i} W\left(X, a_{i}\right)^{k_{i}}\right)=t_{\alpha}(F, H)
$$

where $F$ is the star on $m+1$ nodes, with the endnodes labeled $1, \ldots, m$, and the edge between the center and endnode $i$ replaced by $k_{i}$ parallel edges. Similarly,

$$
\mathrm{E}\left(\prod_{i} W^{\prime}\left(Y, b_{i}\right)^{k_{i}}\right)=t_{\beta}\left(F, H^{\prime}\right) .
$$

These numbers are equal by the hypothesis of the Lemma. This proves that $\lambda_{\alpha}=\lambda_{\beta}^{\prime}$.

Second, we show that $W_{\alpha}(x, y)=W_{\beta}^{\prime}(x, y)$ for almost all $x, y \in[0,1]^{\mathbb{N}}$. It suffices to show that the random variables $Z_{1}=\left(X, Y, W_{\alpha}(X, Y)\right)$ and $Z_{2}=\left(X, Y, W_{\beta}^{\prime}(X, Y)\right)$ (with values from $\left.[0,1]^{\mathbb{N}} \times[0,1]^{\mathbb{N}} \times[0,1]\right)$ have the same distribution, where $X$ and $Y$ are independent points in $\left(\Omega^{\mathbb{N}}, \lambda_{\alpha}\right)$.

We can generate $Z_{1}$ by choosing independent uniform random points $X^{\prime}$ and $Y^{\prime}$ from $\Omega$, and letting $X=\Phi_{\alpha}\left(X^{\prime}\right)$ and $Y=\Phi_{\beta}\left(Y^{\prime}\right)$. Since $\alpha$ is regular, 
we have that

$$
W_{\alpha}(X, Y)=W\left(X^{\prime}, Y^{\prime}\right)
$$

with probability one, and hence

$$
Z_{1}=\left(W\left(X^{\prime}, a_{1}\right), W\left(X^{\prime}, a_{2}\right), \ldots, W\left(Y^{\prime}, a_{1}\right), W\left(Y^{\prime}, a_{2}\right), \ldots, W\left(X^{\prime}, Y^{\prime}\right)\right)
$$

Similarly, we have

$$
Z_{2}=\left(W^{\prime}\left(X^{\prime \prime}, b_{1}\right), W^{\prime}\left(X^{\prime \prime}, b_{2}\right), \ldots, W^{\prime}\left(Y^{\prime \prime}, b_{1}\right), W^{\prime}\left(Y^{\prime \prime}, b_{2}\right), \ldots, W^{\prime}\left(X^{\prime \prime}, Y^{\prime \prime}\right)\right)
$$

where $X^{\prime \prime}$ and $Y^{\prime \prime}$ are independent random points from $\pi^{\prime}$. To prove that $Z_{1}$ and $Z_{2}$ have the same distribution, it again suffices to prove that they have the same mixed moments.

A particular mixed moment is given by nonnegative integers $\left(k_{1}, k_{2}, \ldots\right)$, $\left(l_{1}, l_{2}, \ldots\right.$ ) and $m$ (of which only a finite number is nonzero; say $k_{i}=l_{i}=0$ for $i>n)$. Let us define the multigraph $F$ as follows. $F$ has two unlabeled nodes $v_{x}$ and $v_{y}$, and $n$ further nodes labeled $1, \ldots, n$. We connect $v_{x}$ to $i$ by $k_{i}$ edges, $v_{y}$ to $i$ by $l_{i}$ edges $(i=1, \ldots, n)$, and $v_{x}$ to $v_{y}$ by $m$ edges. Then $\mathrm{E}\left(W\left(X^{\prime}, a_{1}\right)^{k_{1}} \cdots W\left(X^{\prime}, a_{n}\right)^{k_{n}} W\left(Y^{\prime}, a_{1}\right)^{k_{1}} \cdots W\left(Y^{\prime}, a_{n}\right)^{k_{n}} W\left(X^{\prime}, Y^{\prime}\right)\right)=t_{\alpha}(F, H)$. and similarly $\mathrm{E}\left(W^{\prime}\left(X^{\prime \prime}, b_{1}\right)^{k_{1}} \cdots W^{\prime}\left(X^{\prime \prime}, b_{n}\right)^{k_{n}} W^{\prime}\left(Y^{\prime \prime}, b_{1}\right)^{k_{1}} \cdots W^{\prime}\left(Y^{\prime \prime}, b_{n}\right)^{k_{n}} W^{\prime}(X, Y)\right)=t_{\beta}\left(F, H^{\prime}\right)$.

These two numbers are the same by hypothesis. This completes the proof of the Lemma.

To prove Theorem 2.1, we next show:

Lemma 5.3 Let $H=(\Omega, \mathcal{A}, \pi, W)$ and $H^{\prime}=\left(\Omega^{\prime}, \mathcal{A}^{\prime}, \pi^{\prime}, W^{\prime}\right)$ be two Lebesguian graphons such that

$$
t(F, H)=t\left(F, H^{\prime}\right) .
$$


for every multigraph $F$. Then we can couple sequences $\alpha \in \Omega^{\mathbb{N}}$ with sequences $\beta \in \Omega^{\mathbb{N}}$ so that if $\left.\alpha, \beta\right)$ is a sequence from this joint distribution, then

$$
t_{\alpha}(F, H)=t_{\beta}\left(F, H^{\prime}\right)
$$

holds almost surely for every partially labeled multigraph $F$.

Proof. Let $\mathcal{F}_{k}$ be the set of $k$-labeled multigraphs. We define recursively a coupling of sequences $\alpha \in \Omega^{k}$ with sequences $\beta \in \Omega^{\prime k}$ so that $t_{\alpha^{\prime}}(F, H)=t_{\beta^{\prime}}\left(F, H^{\prime}\right)$ holds almost surely for every $F \in \mathcal{F}_{k}$. Let $\left(a_{1}, \ldots, a_{k}\right)$ and $\left(b_{1}, \ldots, b_{k}\right)$ be chosen from this coupled distribution. Consider two random points $X$ from $\pi$ and $Y$ from $\pi^{\prime}$, and the random variables

$$
A=\left(t_{a_{1} \ldots a_{k} X}(F, H): F \in \mathcal{F}_{k+1}\right)
$$

and

$$
B=\left(t_{b_{1} \ldots b_{k} Y}(F, H): F \in \mathcal{F}_{k+1}\right)
$$

with values in $[0,1]^{\mathcal{F}_{k+1}}$. We claim that the variables $A$ and $B$ have the same distribution. It suffices to show that $A$ and $B$ have the same mixed moments. Consider any moment of $A$; in other words, let $F_{1}, \ldots, F_{m} \in \mathcal{F}_{k+1}$, let $q_{1}, \ldots, q_{m}$ be nonnegative integers, and let $F_{i}^{q_{i}}$ be obtained from $F_{i}$ by replacing each edge in $F_{i}$ by $q_{i}$ edges. Then the corresponding moment of $A$ is

$$
\mathrm{E}\left(\prod_{i=1}^{m} t_{a_{1} \ldots a_{k} X}\left(F_{i}, H\right)^{q_{i}}\right)=\mathrm{E}\left(t_{a_{1} \ldots a_{k} X}\left(F_{1}^{q_{1}} \ldots F_{m}^{q_{m}}, H\right)\right)=t_{a_{1} \ldots a_{k}}(F, H),
$$

where the multigraph $F$ is obtained by unlabeling the node labeled $k+1$ in the multigraph $F_{1}^{q_{1}} \ldots F_{m}^{q_{m}}$. Expressing the moments of $B$ in a similar way, we see that they are equal by the induction hypothesis. This proves that $A$ and $B$ have the same distribution.

Using Lemma 6.2 it follows that we can couple the variables $X$ and $Y$ so that $A=B$ with probability 1 . In other words, we can replace $X$ and $Y$ by 
a random variable $\left(X^{\prime}, Y^{\prime}\right) \in \Omega \times \Omega^{\prime}$ so that $X^{\prime}$ has distribution $\pi, Y^{\prime}$ has distribution $\pi^{\prime}$, and their joint distribution satisfies

$$
t_{a_{1} \ldots a_{k} X^{\prime}}(F, H)=t_{b_{1} \ldots b_{k} Y^{\prime}}\left(F, H^{\prime}\right)
$$

for every $F \in \mathcal{F}_{k+1}$ with probability 1 . Thus we have extended the coupling to $\Omega^{k} \times \Omega^{\prime k}$.

It is clear that this sequence of couplings defines a coupling of $\Omega^{\mathbb{N}}$ with $\Omega^{\prime \mathbb{N}}$ as claimed.

\subsection{Conclusion of proofs}

Proof of Theorem 2.1. Part (i) follows easily: if we choose random sequences $(\alpha, \beta)$ from the coupled distribution given by Lemma 5.3, then these sequences will be regular with probability 1 , and so they satisfy the conditions of Lemma 5.2 .

To prove (ii), suppose that $H=(\Omega, \mathcal{A}, \pi, W)$ and $H^{\prime}=\left(\Omega^{\prime}, \mathcal{A}^{\prime}, \pi^{\prime}, W^{\prime}\right)$ satisfy (4) for every simple graph $F$. By Corollary 3.3, we can find twinfree Lebesguian graphons $G=(\Gamma, \mathcal{B}, \rho, U)$ and $G^{\prime}=\left(\Gamma^{\prime}, \mathcal{B}^{\prime}, \rho^{\prime}, U^{\prime}\right)$ and weak isomorphisms $\phi$ and $\phi^{\prime}$ from $H$ and $H^{\prime}$ to $G$ and $G^{\prime}$, respectively. It follows by Theorem 2.1(i) that the $G$ and $G^{\prime}$ are isomorphic mod 0 , so in particular $U=\left(U^{\prime}\right)^{\psi^{\prime}}$ almost everywhere for some measure preserving map $\psi^{\prime}: \Gamma \rightarrow \Gamma^{\prime}$. Defining $\psi: \Omega \rightarrow \Gamma^{\prime}$ by $\psi(x)=\psi^{\prime}(\phi(x))$, we conclude that $W=\left(U^{\prime}\right)^{\psi}$ almost everywhere. The maps $\psi$ and $\phi^{\prime}$ are measure preserving from the completions $\bar{H}$ and $\overline{H^{\prime}}$ into $G^{\prime}$.

Proof of Corollary 2.2. The equivalence of (a), (b) and (c) follows by Theorem 2.1(ii) and the fact that a function which is measurable with respect to the completion of $\mathcal{L} \times \mathcal{L}$ is almost everywhere equal to a function which is measurable with respect to $\mathcal{L} \times \mathcal{L}$. In the proof of $(\mathrm{c})$, Theorem 2.1 may give a graphon containing atoms, but it is easy to replace these atoms by intervals of appropriate length.

To prove that $(\mathrm{c}) \Longrightarrow(\mathrm{e})$, assume that $\varphi, \psi$ and $U$ exist as in (c). Let $X, X^{\prime} \in[0,1]$ be independent random points from the uniform distribution 
$\lambda$ on $[0,1]$. Since $\varphi$ and $\psi$ are measure preserving, $\varphi(X)$ and $\psi(Y)$ have the same distribution, and hence by Lemma 6.2 there is a coupling measure $\gamma$ on $[0,1] \times[0,1]$ with marginals $\lambda$ such that if $\left(X, X^{\prime}\right)$ is a random sample

from $\gamma$, then $\varphi(X)=\psi\left(X^{\prime}\right)$ with probability 1 . So if $\left(X, X^{\prime}\right)$ and $\left(Y, Y^{\prime}\right)$ are independent random points from $\gamma$, then

$$
W(X, Y)=U(p h i(X), \operatorname{phi}(Y))=U^{\prime}\left(p s i\left(X^{\prime}\right), \operatorname{psi}\left(Y^{\prime}\right)\right)=W^{\prime}\left(X^{\prime}, Y^{\prime}\right) .
$$

To prove that $(\mathrm{e}) \Longrightarrow(\mathrm{d})$, consider the projections $\Phi, \Psi:[0,1]^{2} \rightarrow[0,1]$ defined by $\Phi\left(x, x^{\prime}\right)=x$ and $\Psi\left(x, x^{\prime}\right)=x^{\prime}$. Then

$$
W^{\Phi}\left(\left(X, X^{\prime}\right),\left(Y, Y^{\prime}\right)\right)=W(X, Y)
$$

and

$$
\left(W^{\prime}\right)^{\Psi}\left(\left(X, X^{\prime}\right),\left(Y, Y^{\prime}\right)\right)=W^{\prime}\left(X^{\prime}, Y^{\prime}\right)
$$

Thus, $W^{\Phi}=\left(W^{\prime}\right)^{\Psi}$ almost everywhere. Furthermore, $\Phi$ and $\Psi$ are measure preserving if we consider the coupling measure $\gamma$ on $[0,1]$.

Since the completion of $\left([0,1]^{2}, \mathcal{L}_{2}, \gamma\right)$ is a Lebesgue space, we can find a measure preserving map $\rho:([0,1], \lambda) \rightarrow\left([0,1]^{2}, \gamma\right)$. Setting $\varphi=\Phi \circ \rho$ and $\psi=\Psi \circ \rho$, we obtain the desired measure preserving maps $\varphi, \psi:[0,1] \rightarrow[0,1]$ such that $W^{\varphi}=\left(W^{\prime}\right)^{\psi}$ almost everywhere.

Finally, $(\mathrm{d}) \Rightarrow(\mathrm{a})$ is trivial.

\section{Acknowledgement}

We are grateful to Miklós Laczkovich, Ron Peled, Yuval Peres and Oded Schramm for many useful discussions on the topic of this paper, and to Kati Vesztergombi and Svante Janson for carefully reading an earlier version and suggesting several improvements. 


\section{References}

[1] N. Alon, W. Fernandez de la Vega, R. Kannan and M. Karpinski: Random sampling and approximation of MAX-CSPs, J. Comput. System Sci. 67 (2003), 212-243.

[2] C. Borgs, J. Chayes, L. Lovász, V.T. Sós, K. Vesztergombi, unpublished, 2004.

[3] C. Borgs, J. Chayes, L. Lovász, V.T. Sós, K. Vesztergombi: Counting graph homomorphisms, in: Topic in Discrete Mathematics (Klazar, Kratochvil, Loebl, Matousek, Thomas, Valtr, eds.) Springer, BerlinHeidelberg (2006), 315-371.

[4] C. Borgs, J. Chayes, L. Lovász, V.T. Sós, K. Vesztergombi: Convergent Sequences of Dense Graphs I: Subgraph Frequencies, Metric Properties and Testing, preprint (2006), http://research.microsoft.com/ borgs/Papers/ConvMetric.pdf

[5] C. Borgs, J.T. Chayes, L. Lovász, V.T. Sós, and K. Vesztergombi: Convergent Graph Sequences II. H-Colorings, Statistical Physics and Quotients, manuscript (2006)

[6] P. Erdös, L. Lovász, J. Spencer: Strong independence of graphcopy functions, in: Graph Theory and Related Topics, Academic Press, 165172.

[7] Feller, W.: An Introduction to Probability Theory and its Applications, Vol. II, Second edition, Wiley, NewYork, (1971).

[8] M. Freedman, L. Lovász, A. Schrijver: Reflection positivity, rank connectivity, and homomorphism of graphs Journal of The American Mathematical Society (to appear)

[9] O. Goldreich, S. Goldwasser and D. Ron: Property testing and its connection to learning and approximation, J. ACM 45 (1998), 653-750. 
[10] P.R. Halmos: Measure Theory, Graduate Texts in Mathematics 18, Springer, New York, Heidelberg, Berlin (1991).

[11] L. Lovász and B. Szegedy: Limits of dense graph sequences, Microsoft Research Technical Report TR-2004-79.

[12] L. Lovász, B. Szegedy: Szemerédi's Lemma for the analyst, Microsoft Research Technical Report MSR-TR-2005-90, ftp://ftp.research.microsoft.com/pub/tr/TR-2005-90.pdf

[13] V. A. Rohlin: On the fundamental ideas of measure theory, Translations of the American Mathematical Society, Series 1, Vol. 10, 1-54 (1962). The Russian original appeared in Mat. Sb. 25, 107-150 (1949).

[14] D. Williams: Probability with Martingales, Cambridge University Press, (1991).

\section{Appendix: Moments and coupling of prob- ability distributions}

In this section we prove some probability theory lemmas, that are "well known" but not easy to reference. We start with the fact that if two vector valued random variables have the same mixed moments, then they have the same distribution (cf Feller [7], Problem XV.9.21.).

Lemma 6.1 Let $(\Omega, \mathcal{A}, \pi)$ and $\left(\Omega^{\prime}, \mathcal{A}^{\prime}, \pi^{\prime}\right)$ be probability spaces, and let $f: \Omega \rightarrow[0,1]^{\mathbb{N}}$ and $g: \Omega^{\prime} \rightarrow[0,1]^{\mathbb{N}}$ be measurable functions, with $f(x)=\left(f_{1}(x), f_{2}(x), \ldots\right)$ and $g(y)=\left(g_{1}(y), g_{2}(y), \ldots\right)$. If

$$
\int f_{1}(x)^{k_{1}} \ldots f_{n}(x)^{k_{n}} d \pi(x)=\int g_{1}(y)^{k_{1}} \ldots g_{n}(y)^{k_{n}} d \pi^{\prime}(y)
$$

for every finite sequence of nonnegative integers $k_{1}, \ldots, k_{n}$, then $\pi\left(f^{-1}(B)\right)=$ $\pi^{\prime}\left(g^{-1}(B)\right)$ for every Borel set $B \subseteq[0,1]^{\mathbb{N}}$. 
Proof. It suffices to prove that $\pi\left(f^{-1}(B)\right)=\pi^{\prime}\left(g^{-1}(B)\right)$ for every Borel set of the form $B=I_{1} \times I_{2} \times \ldots I_{n} \times[0,1] \times \ldots$, where $I_{1}, \ldots, I_{n}$ are intervals. Let $p_{j, m}(x)$ be a polynomial that approximates the indicator function $\mathbf{1}_{I_{j}}$ on $[0,1]$ in $L_{1}$ with error less than $1 / m(j=1, \ldots, n)$. Then

$$
\begin{aligned}
\int_{\Omega} p_{1, m}\left(f_{1}(x)\right) \cdots p_{n, m}\left(f_{n}(x)\right) d x & \longrightarrow \int_{\Omega} \mathbf{1}_{I_{1}}\left(f_{1}(x)\right) \cdots \mathbf{1}_{I_{n}}\left(f_{n}(x)\right) d x \\
& =\int_{f^{-1}(B)} 1 d x=\pi\left(f^{-1}(B)\right) \quad(m \rightarrow \infty) .
\end{aligned}
$$

Similarly,

$$
\int_{\Omega} p_{1, m}\left(g_{1}(x)\right) \cdots p_{n, m}\left(g_{n}(x)\right) d x \longrightarrow \pi\left(g^{-1}(B)\right) \quad(m \rightarrow \infty) .
$$

But the left hand sides of these two relations are equal for all $m$, which proves the Lemma.

We need the following natural fact about coupling.

Lemma 6.2 Assume that $(\Omega, \mathcal{A}, \pi)$ and $\left(\Omega^{\prime}, \mathcal{A}, \pi^{\prime}\right)$ are Lebesgue spaces, and $(\Gamma, \mathcal{B}, \rho)$, a countably generated separating space. Let $f: \Omega \rightarrow \Gamma$ and $g: \Omega^{\prime} \rightarrow \Gamma$ be measure preserving maps. Then there exists a coupling $\nu$ of $(\Omega, \mathcal{A}, \pi)$ and $\left(\Omega^{\prime}, \mathcal{A}, \pi^{\prime}\right)$ such that

$$
\nu\{(x, y): f(x)=g(y)\}=1
$$

Proof. For $A \in \mathcal{A}$, consider the measure $\lambda^{A}(B)=\pi\left(A \cap f^{-1}(B)\right)$ defined for $B \in \mathcal{B}$, and its Radon-Nikodym derivative $f^{A}=d \lambda^{A} / d \rho$. Since $\lambda^{A} \leq$ $\pi\left(f^{-1}(B)\right)=\rho(B)$, this derivative exists, and $0 \leq f^{A} \leq 1$ almost everywhere. Furthermore, $f^{\emptyset}=0$ and $f^{\Omega}=1$ almost everywhere.

Similarly, for $C \in \mathcal{A}^{\prime}$, define $\mu^{C}(B)=\pi^{\prime}\left(C \cap g^{-1}(B)\right)$ and $g^{C}=d \mu^{C} / d \rho$. Finally, let

$$
\nu(A \times C)=\int f^{A} g^{C} d \rho
$$


Clearly

$$
\nu(A \times C) \leq \int f^{A} d \rho=\pi(A),
$$

and similarly $\nu(A \times C) \leq \pi^{\prime}(C)$. Hence in particular $\nu(A \times C)=0$ if either $\pi(A)=0$ or $\pi^{\prime}(C)=0$.

Claim 2 If $A_{i} \in \mathcal{A}, C_{i} \in \mathcal{A}^{\prime}(i \in I)$ and the sets $A_{i} \times C_{i}$ form a (finite or countably infinite) partition of $A \times C\left(A \in \mathcal{A}, C \in \mathcal{A}^{\prime}\right)$, then $\sum_{i} \nu\left(A_{i} \times C_{i}\right)=$ $\nu(A \times C)$.

It is easy to see that if $A_{1}, A_{2} \in \mathcal{A}$ are disjoint sets and $A=A_{i} \cup A_{2}$, then $f^{A_{1}}+f^{A_{2}}=f^{A}$ almost everywhere. It follows that for every $C \in \mathcal{A}^{\prime}$, we have $\nu\left(A_{1} \times C\right)+\nu\left(A_{2} \times C\right)=\nu(A \times C)$. This implies by standard arguments that the claim holds if $|I|$ is finite. This in turn implies that $\nu$ extends to a finitely additive measure on the algebra $\mathcal{F}$ of sets that can be written as the union of a finite number of product sets $A \times C\left(A \in \mathcal{A}, C \in \mathcal{A}^{\prime}\right)$.

In the case of infinite $|I|$, it follows that $\sum_{i} \nu\left(A_{i} \times C_{i}\right) \leq \nu(A \times C)$; in fact, for every finite $J \subseteq I$, we have $\cup_{i \in J} A_{i} \times C_{i} \subseteq A \times C$, and hence by the finite additivity of $\nu$, we have

$$
\sum_{i \in J} \nu\left(A_{i} \times C_{i}\right)=\nu\left(\cup_{i \in J} A_{i} \times C_{i}\right) \leq \nu(A \times C) .
$$

Since this holds for every finite subset $J$ of $I$, it also holds for $I$.

Suppose that there is a partition where $\left\{A_{i} \times C_{i}: i=1 \in \mathbb{N}\right\}$ of $A \times C$ and an $\varepsilon>0$ for which

$$
\sum_{i} \nu\left(A_{i} \times C_{i}\right)<\nu(A \times C)-4 \varepsilon
$$

on a set $B$ of positive measure. Now we use that $(\Omega, \mathcal{A}, \pi)$ and $\left(\Omega^{\prime}, \mathcal{A}, \pi^{\prime}\right)$ are Lebesgue spaces, so we may assume that they are intervals $[0, a]$ and $[0, b]$ respectively, together with a countable set of atoms. Thinking of the atoms as converging to $a$ from above, we have a compact topology on them. For every $i$, we can find an open sets $U_{i} \supseteq A_{i}$ and $V_{i} \supseteq C_{i}$ such that $\pi\left(U_{i}\right) \leq \pi\left(A_{i}\right)+\varepsilon 2^{-i}$ 
and $\pi^{\prime}\left(V_{i}\right) \leq \pi^{\prime}\left(C_{i}\right)+\varepsilon / 2^{i}$. Also, we can find closed sets $U \subseteq A$ and $V \subseteq C$ such that $\pi(U) \geq \pi(A)-\varepsilon$ and $\pi^{\prime}(V) \geq \pi^{\prime}(C)-\varepsilon$. Then

$$
\begin{aligned}
\nu\left(U_{i} \times V_{i}\right) & \leq \nu\left(A_{i} \times C_{i}\right)+\nu\left(\left(U_{i} \backslash A_{i}\right) \times C_{i}\right)+\nu\left(U_{i} \times\left(V_{i} \backslash C_{i}\right)\right) \\
& \leq \nu\left(A_{i} \times C_{i}\right)+\pi\left(U_{i} \backslash A_{i}\right)+\pi^{\prime}\left(V_{i} \backslash C_{i}\right) \leq \nu\left(A_{i} \times C_{i}\right)+2 \varepsilon 2^{-i} .
\end{aligned}
$$

It follows similarly that

$$
\nu(U \times V) \geq \nu(A \times C)-2 \varepsilon .
$$

Hence

$$
\sum_{i} \nu\left(U_{i} \times V_{i}\right) \leq \sum_{i} \nu\left(A_{i} \times C_{i}\right)+2 \varepsilon<\nu(A \times C)-2 \varepsilon \leq \nu(U \times V) .
$$

The open sets $U_{i} \times V_{i}$ cover the compact set $U \times V$, and so a finite number of them also covers. But the contradicts the finite additivity of $\nu$ which we already established.

Claim 3 The setfunction $\nu$ extends to a measure on $\mathcal{A} \times \mathcal{A}^{\prime}$.

We have seen already that $\nu$ extends to $\mathcal{F}$; it follows by Claim 2 that this extension is $\sigma$-additive. Thus the Claim follows by the Measure Extension Theorem.

Define $\Delta=\left\{(x, y) \in \Omega \times \Omega^{\prime}: f(x)=g(y)\right\}$. To complete the proof of the Lemma, we want to prove that $\nu$ is a coupling between $(\Omega, \mathcal{A}, \pi)$ and $\left(\Omega^{\prime}, \mathcal{A}, \pi^{\prime}\right)$ (which is trivial), and that $\nu\left(\Omega \times \Omega^{\prime} \backslash \Delta\right)=0$. Let $\mathcal{S} \subseteq \mathcal{B}$ be a countable family separating the elements of $\Gamma$. Then

$$
\Omega \times \Omega^{\prime} \backslash \Delta=\bigcup_{S \in \mathcal{S}} f^{-1}(S) \times g^{-1}(\Gamma \backslash S) \cup \bigcup_{S \in \mathcal{S}} f^{-1}(\Gamma \backslash S) \times g^{-1}(S) .
$$

Consider any term here, say $f^{-1}(S) \times g^{-1}(\Gamma \backslash S)=A \times C$. Then

$$
\nu(A \times C)=\int f^{A} g^{C} d \rho=\int_{S}+\int_{\Gamma \backslash S} .
$$


Here

$$
\int_{S} f^{A} g^{C} d \rho \leq \int_{S} g^{C} d \rho=\mu^{C}(S)=\pi^{\prime}\left(g^{-1}(\Gamma \backslash S) \cap g^{-1}(S)\right)=0,
$$

and similarly

$$
\int_{\Omega \backslash S} f^{A} g^{C} d \rho=0 .
$$

This proves that $\nu\left(\Omega \times \Omega^{\prime} \backslash \Delta\right)=0$. 